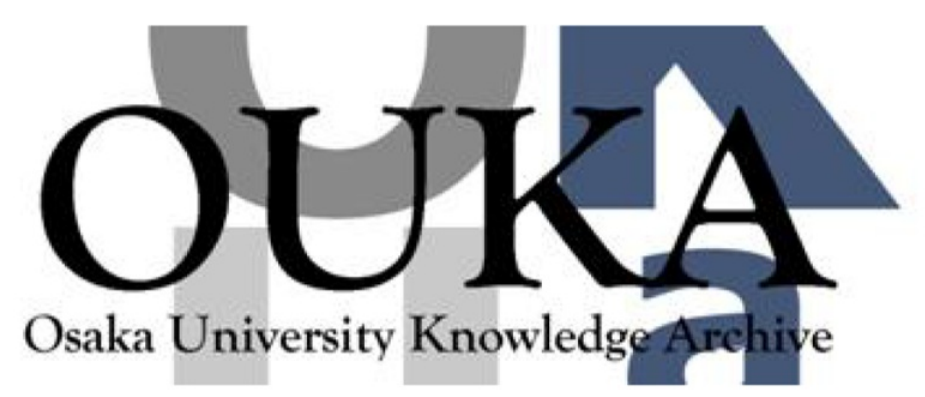

\begin{tabular}{|c|l|}
\hline Title & $\begin{array}{l}\text { Heavy Higgs boson at the Tevatron and the LHC } \\
\text { in universal extra dimension models }\end{array}$ \\
\hline Author(s) & $\begin{array}{l}\text { Nishiwaki, Kenji; Oda, Kinya; Okuda, Naoya et } \\
\text { al. }\end{array}$ \\
\hline Citation & Physical Review D. 85(3) p. 035026 \\
\hline Issue Date & $2012-02-23$ \\
\hline oaire:version VoR \\
\hline URL & https://hdl.handle.net/11094/78756 \\
\hline rights & ○ 2012 American Physical Society \\
\hline Note & \\
\hline
\end{tabular}

Osaka University Knowledge Archive : OUKA

https://ir. Library. osaka-u. ac. jp/

Osaka University 


\title{
Heavy Higgs boson at the Tevatron and the LHC in universal extra dimension models
}

\author{
Kenji Nishiwaki (西脇健二), ${ }^{1,2, *}$ Kin-ya Oda (尾田欣也), ${ }^{3, \dagger}$ \\ Naoya Okuda (奥田直也), ${ }^{3, *}$ and Ryoutaro Watanabe (渡邊諒太郎) $)^{3,8}$ \\ ${ }^{1}$ Department of Physics, Kobe University, Kobe 657-8501, Japan \\ ${ }^{2}$ Harish-Chandra Research Institute, Chhatnag Road, Jhusi, Allahabad 211 019, India \\ ${ }^{3}$ Department of Physics, Osaka University, Osaka 560-0043, Japan
}

(Received 10 August 2011; published 23 February 2012)

\begin{abstract}
Universal extra dimension (UED) models tend to favor a distinctively heavier Higgs mass than in the standard model and its supersymmetric extensions when the Kaluza-Klein (KK) scale is not much higher than the electroweak one, which we call the weak scale UED, in order to cancel the KK-top contributions to the $T$ parameter. Such a heavy Higgs, whose production through the gluon fusion process is enhanced by the KK-top loops, is a fairly model independent prediction of the weak scale UED models regardless of the brane-localized mass structure at the ultraviolet cutoff scale. We study its cleanest possible signature, the Higgs decay into a $Z$ boson pair and subsequently into four electrons and/or muons, in which all the four-momenta of the final states can be measured and both the $Z$ boson masses can be checked. We have studied the Higgs mass, $500 \mathrm{GeV}$ (and also $700 \mathrm{GeV}$ with $\sqrt{s}=14 \mathrm{TeV}$ ), and have found that we can observe significant resonance with the integrated luminosity $10 \mathrm{fb}^{-1}$ for six-dimensional UED models.
\end{abstract}

DOI: 10.1103/PhysRevD.85.035026

PACS numbers: 14.80.Ec, 04.50.Cd, 11.10.Kk, 14.80.Rt

\section{INTRODUCTION}

The universal extra dimension (UED) scenario [1], in which all the standard model (SM) fields propagate in the bulk of compactified extra dimension(s), is an attractive possibility whose simplest five-dimensional realization on orbifold $S^{1} / Z_{2}$, the minimal UED model (mUED), may account for the existence of dark matter as the lightest Kaluza-Klein particle (LKP) [2] and can give a loose gauge coupling unification at around $30 \mathrm{TeV}$ [3]. See also Refs. [4,5] for review on mUED. For the mUED, the latest analysis including the effects from the second KaluzaKlein $(\mathrm{KK})$ resonances gives the preferred $\mathrm{KK}$ scale at around $M_{\mathrm{KK}} \sim 1.3 \mathrm{TeV}$ [6]. As is mentioned in [2,6], this result strongly depends on the brane-localized mass structure, which is assumed to be vanishing at the UV cutoff scale [7] in mUED.

One of the most important signatures to establish the model would be the direct search of KK resonances at the CERN Large Hadron Collider (LHC). See Refs. [8-14] for mUED and Refs. [15-21] for 6D UED models. We note that some of them also pertain to the International Linear Collider (ILC); see also Refs. [22-27]. The LHC already puts a lower bound on the KK scale for mUED as $M_{\mathrm{KK}} \gtrsim$ $500 \mathrm{GeV}$ at the $95 \%$ C.L. from an $M_{T 2}$ analysis of cascade decay of the first KK particles into the LKP [14,28]. It is noted that bounds on mUED [29] and $T^{2} / Z_{4}$ UED [30] from $b \rightarrow s \gamma$ processes claim $M_{\mathrm{KK}} \gtrsim 600$ and $650 \mathrm{GeV}$,

\footnotetext{
*nishiwaki@hri.res.in

†odakin@phys.sci.osaka-u.ac.jp

‡okuda@het.phys.sci.osaka-u.ac.jp

§ryoutaro@het.phys.sci.osaka-u.ac.jp
}

respectively. Again, all the above bounds strongly depend on the KK mass splitting and flavor mixing patterns and hence on the boundary mass structure. ${ }^{1}$ In particular, we cannot see a decay product unless there is enough mass splitting among the first KK modes so that it becomes sufficiently energetic. In this paper, we present a complementary signal that is insensitive to such detailed boundary structure.

In the SM, the electroweak data constrain the Higgs mass to be $M_{H} \lesssim 170 \mathrm{GeV}$ at the $95 \%$ C.L.; see, e.g., [32,33]. On the contrary, mUED prefer heavier Higgs when the KK scale is not much higher than the electroweak scale $v_{\mathrm{EW}} \simeq 246 \mathrm{GeV}$, namely, the $\mathrm{KK}$ scale should be $M_{\mathrm{KK}} \gtrsim 800 \mathrm{GeV}\left(300 \mathrm{GeV} \lesssim M_{\mathrm{KK}} \lesssim 400 \mathrm{GeV}\right)$ at the 95\% C.L. for $M_{H}=115(700 \mathrm{GeV})$ [34-36]. We note that this is a fairly model independent feature of a general UED model since KK-top modes always contribute positively to the $T$ parameter and such an effect requires a heavy Higgs in order to cancel these KK-top contributions by the ordinary negative $\log M_{H}$ dependence. In this paper, we call such a natural UED model without a big mass splitting among electroweak, Higgs and KK scales: $M_{H} \sim M_{\mathrm{KK}} \sim \mathcal{O}\left(10^{2} \mathrm{GeV}\right)$, the weak scale UED model. Concretely, we will pick up the cases: $M_{H}=330,500$, and $700 \mathrm{GeV}$. To summarize, the existence of a heavy Higgs is a model independent prediction of the weak scale

\footnotetext{
${ }^{1}$ A $95 \%$ C.L. bound on the KK scale $M_{\mathrm{KK}}>961 \mathrm{GeV}$ is put on a "UED" model, assuming the existence of large additional extra dimensions compactified with a radius of order $\mathrm{eV}^{-1}$, in which SM fields cannot propagate, so that the LKP decays into KK gravitons [31]. In this paper, we do not assume such additional large extra dimensions.
} 
UED models in contrast to the relic abundance of dark matter and the cascade decay signature of the KK particles that are dependent on the detailed boundary mass structure at the UV cutoff scale of the higher-dimensional gauge theory.

In five- and six-dimensional UED models, the Higgs production cross section via the gluon fusion process is enhanced by the KK-top loops [37-39]. In this paper, we analyze its cleanest possible signature, the Higgs decay, into a $Z$ boson pair and subsequently into four electrons and/or muons, in which all the four-momenta of the final states can be measured and both the $Z$ boson masses can be checked.

In Sec. II, we review the relevant part of all the known 5D and 6D UED models to the Higgs production process via the gluon fusion through the KK-top loops. Considered models are the 5D mUED model on $S^{1} / Z_{2}$ [1], the Dirichlet Higgs (DH) model on an interval $[40,41]$, the 6D $T^{2}$-based models on $T^{2} / Z_{2}$ [1], $T^{2} /\left(Z_{2} \times Z_{2}^{\prime}\right)$ [42], $T^{2} / Z_{4}$ [43,44], $R P^{2}$ [45], and the 6D $S^{2}$-based models on the projective sphere (PS) [46], $S^{2}$ (see Sect. II C 2), $S^{2} / Z_{2}$ [47]. ${ }^{2}$ In Sec. III, we present the concrete computation of the process. The cross sections for the $\mathrm{DH}, T^{2} / Z_{2}$, $T^{2} /\left(Z_{2} \times Z_{2}^{\prime}\right), R P^{2}$, and $S^{2}$ UED models are newly obtained. We also review the estimation of the UV cutoff scale in the $T^{2}$-based geometry [48] and extend it to the $S^{2}$-based one. In Sec. IV, we show our numerical results. The last section is for summary and discussions. In Appendix A, we present the relevant Feynman rules for our computation in the DH model. In Appendix B we explain our estimation of the UV cutoff scale for $6 \mathrm{D}$ UED models, based on the renormalization group equation (RGE) analysis in the renormalizable KK picture. In Appendix $\mathrm{C}$, we review the way to take into account the width in the amplitude and justify our approximation.

\section{REVIEW ON KNOWN 5D AND 6D UED MODELS}

In this section, we give a brief review on various UED models. Readers who are not interested in the details of these models may skip this section. In the first part of this section, we briefly review the 5D minimal UED model on $S^{1} / Z_{2}$ [1] and Dirichlet Higgs model on an interval [40,41]. The remaining of the section is devoted to an overview of various types of 6D UED models.

\section{A. 5D UED models}

\section{Minimal UED model on $S^{1} / Z_{2}$}

First we review the 5D UED model [1]. The matter contents of the model are the same as those of the SM,

\footnotetext{
${ }^{2}$ In [46] the terminology "real projective plane" is employed for the compactified space, the sphere with its antipodal points being identified. In order to distinguish [46] from [45], we call the former the projective sphere.
}

but they are living in the bulk of flat five-dimensional space, compactified on the orbifold $S^{1} / Z_{2}$. The action $S$ is written down as

$S=\int d^{4} x \int_{-\pi R}^{\pi R} d y\left[\mathcal{L}_{\text {bulk }}+\delta(y) \mathcal{L}_{0}+\delta(y-\pi R) \mathcal{L}_{\pi R}\right]$.

Usually when one says mUED model, it is implied that all the boundary masses are zero at the UV cutoff scale and are generated through radiative corrections [7]. Hereafter, when we say mUED model, we do not assume any boundary mass structure and concentrate on the signal that is independent of it. In particular, we do not include the constraints from the direct KK search [14,28] and from the relic abundance of the LKP [6] that are dependent on the KK mass splitting pattern.

The $Z_{2}$ twist conditions on the bulk SM fields are indicated as

$$
\begin{gathered}
\mathcal{B}_{\mu}(x,-y)=\mathcal{B}_{\mu}(x, y), \quad \mathcal{B}_{5}(x,-y)=-\mathcal{B}_{5}(x, y), \\
\mathcal{W}_{\mu}(x,-y)=\mathcal{W}_{\mu}(x, y), \quad \mathcal{W}_{5}(x,-y)=-\mathcal{W}_{5}(x, y), \\
\mathcal{G}_{\mu}(x,-y)=G_{\mu}(x, y), \quad G_{5}(x,-y)=-\mathcal{G}_{5}(x, y), \\
L(x,-y)=\gamma^{5} L(x, y), \quad E(x,-y)=-\gamma^{5} E(x, y), \\
Q(x,-y)=\gamma^{5} Q(x, y), \quad U(x,-y)=-\gamma^{5} U(x, y), \\
D(x,-y)=-\gamma^{5} D(x, y),
\end{gathered}
$$

and

$$
\Phi(x,-y)=\Phi(x, y),
$$

where $x$ and $y\left(=x^{5}\right)$ denote four- and extra-dimensional coordinates, respectively. ${ }^{3}$ We can see that the wanted zero modes remain after the twist (2)-(4). There are fixed points of the $Z_{2}$ orbifolding at $y=0, \pi R$. If the boundary Lagrangians at $y=0, \pi R$ are equal at the UV cutoff scale, there remains an additional accidental symmetry under the reflection $\frac{\pi R}{2}-y \rightarrow \frac{\pi R}{2}+y$, called the KK parity, which ensures the stability of the LKP and makes it a dark matter candidate.

The gauge and Yukawa interactions for the KK-top quarks, which we need for later calculation, are

$$
\begin{aligned}
& \mathcal{L}_{\mathrm{KK} \text { top }}=-i g_{4 s} \sum_{n=1}^{\infty}\left[\begin{array}{ll}
\bar{t}_{1} & \bar{t}_{2}
\end{array}\right]^{(n)} \gamma^{\mu} \mathcal{G}_{\mu}^{(0)}\left[\begin{array}{l}
t_{1} \\
t_{2}
\end{array}\right]^{(n)} \\
& -\frac{m_{t}}{v_{\mathrm{EW}}} H^{(0)} \sum_{n=1}^{\infty}\left[\begin{array}{ll}
\bar{t}_{1} & \bar{t}_{2}
\end{array}\right]^{(n)} \\
& \times\left[\begin{array}{cc}
\sin 2 \alpha^{(n)} & -\gamma^{5} \cos 2 \alpha^{(n)} \\
\gamma^{5} \cos 2 \alpha^{(n)} & \sin 2 \alpha^{(n)}
\end{array}\right]\left[\begin{array}{l}
t_{1} \\
t_{2}
\end{array}\right]^{(n)},
\end{aligned}
$$

where $g_{4 s}=g_{s} / \sqrt{2 \pi R}$ is a dimensionless $4 \mathrm{D} S U(3)_{C}$ coupling constant and $v_{\mathrm{EW}} \simeq 246 \mathrm{GeV}$ is the 4D Higgs vacuum expectation value which appears after the $\mathrm{KK}$

\footnotetext{
${ }^{3} \mathrm{We}$ follow the metric and spinor conventions of [49].
} 
expansion; $G^{(0)}\left(H^{(0)}\right)$ shows the zero-mode gluon (zero-mode physical Higgs); $t_{1}^{(n)}$ and $t_{2}^{(n)}$ are mass eigenstates of $n$th KK-top quarks and each mixing angle $\alpha^{(n)}$ is determined to be $\cos 2 \alpha^{(n)}=m_{(n)} / \sqrt{m_{t}^{2}+m_{(n)}^{2}}, \sin 2 \alpha^{(n)}=$ $m_{t} / \sqrt{m_{t}^{2}+m_{(n)}^{2}}$, with $m_{(n)}:=n / R$. Each $\mathrm{KK}$ state is twofold degenerate and $n$th KK-top mass is

$$
m_{t,(n)}=\sqrt{m_{t}^{2}+m_{(n)}^{2}} .
$$

KK tops give the dominant contribution to the gluon fusion process due to their large Yukawa coupling to the Higgs. We note that $\gamma^{5}$ is put in Eq. (5) merely to arrange the sign of both the KK masses positive.

\section{Dirichlet Higgs model}

The Dirichlet Higgs model is defined on an interval: $0 \leq y \leq \pi R$. The action $S$ is as follows:

$S=\int d^{4} x \int_{0}^{\pi R} d y\left[\mathcal{L}_{\text {bulk }}+\delta(y) \mathcal{L}_{0}+\delta(y-\pi R) \mathcal{L}_{\pi R}\right]$,

where $R$ is a radius of the extra spacial direction. The structure of the bulk Lagrangian, covariant derivatives and field strength of gauge bosons are the same as that of the mUED model. There is no difference between the matter contents of this model and those of the mUED model. As in the mUED model, we neglect the possible boundary interactions in this paper. The zero-mode sector of the UED on an interval becomes the same as that of the mUED on the orbifold $S^{1} / Z_{2}$ when we choose the boundary conditions for the SM degrees of freedom $\Psi^{N}=G_{\mu}, \mathcal{W}_{\mu}, \mathcal{B}_{\mu} ; L_{L}, Q_{L} ; E_{R}, U_{R}, D_{R}$ to be Neumann (at $y=0$ and $\pi R$ ):

$$
\partial_{5} \Psi^{N}(x ; 0)=\partial_{5} \Psi^{N}(x, \pi R)=0,
$$

and for other non-SM modes $\Psi^{D}=\mathcal{B}_{5}, \mathcal{W}_{5} ; L_{R}, Q_{R}$; $E_{L}, U_{L}, D_{L}$ to be Dirichlet:

$$
\Psi^{D}(x, 0)=\Psi^{D}(x, \pi R)=0 .
$$

We note that mode functions with Dirichlet and Neumann boundary conditions are not orthogonal to each other, unlike the orbifolding on $S^{1} / Z_{2}{ }^{4}$ Kinetic terms turn out to be diagonal even though the expansion is not orthonormal. We can explicitly check that the nonorthogonality does not lead to extra mixing for spinors even after the electroweak symmetry breaking (EWSB) because nonorthogonal terms drop out due to the 4D chirality.

If we had put the Neumann condition on the Higgs, we would get exactly the same zero-mode sector as in the mUED model on $S^{1} / Z^{2}$. In the Drichlet Higgs model on interval, the EWSB is caused by a nonzero Dirichlet

\footnotetext{
${ }^{4}$ In other words the KK mass-squared operator $\partial_{5}^{2}$ is not Hermitian in this setup, though the kinetic term is still positive definite.
}

boundary condition on the $S U(2)_{W}$-doublet Higgs field $[40,41]$. We assume that the KK parity is respected by the boundary conditions on the Higgs field too. The advantage of the Dirichlet EWSB is that we do not need to assume the negative mass squared in the bulk Lagrangian nor the quartic coupling which is a higher-dimensional operator in $5 \mathrm{D}$. Throughout this paper, we consider the minimal case: $\mathcal{V}(\Phi)=0$. We list the necessary Feynman rules in Appendix A.

\section{B. 6D UED models based on $T^{2}$}

We consider a gauge theory on six-dimensional spacetime $M^{4} \times T^{2}$, which is a direct product of the fourdimensional Minkowski spacetime $M^{4}$ and two-torus $T^{2}$ : $0 \leq y \leq 2 \pi R_{y}, 0 \leq z \leq 2 \pi R_{z}$. We assume that the two radii of $T^{2}$ have the same value $R=R_{y}=R_{z}$ for simplicity. ${ }^{5}$

When we use a 6D Weyl spinor for 6D UED model construction, there is a constraint on the choice of $6 \mathrm{D}$ chiralities. The origin of this constraint is the cancellation of $6 \mathrm{D}$ gravitational and $S U(2)_{L}$ global anomalies that cannot be removed by use of the Green-Schwarz mechanism. This constraint requires the number of matter generation to be (multiples of) three [51]. A suitable choice of the $6 \mathrm{D}$ chirality for a single matter generation is as follows:

$$
\left(Q_{+}, U_{-}, D_{-} ; L_{+}, E_{-}, N_{-}\right) \text {, }
$$

where the \pm suffixes represent the $6 \mathrm{D}$ chirality of each field. The number of d.o.f. of the 6D Weyl fermion is 4, the same as that of a 4D Dirac fermion. Therefore, we can construct 6D UED models on $T^{2}$ following the orbifolding method of the 5D UED model. We have several options for the orbifolding to realize the SM chiral fermions in the zero-mode sector of (10). Let us review them in turn. The range for KK summation is listed in Table II.

\section{Orbifold $T^{2} / Z_{2}, T^{2} /\left(Z_{2} \times Z_{2}^{\prime}\right)$, and $T^{2} / Z_{4}$}

We consider $T^{2} / Z_{2}$ [1], $T^{2} /\left(Z_{2} \times Z_{2}^{\prime}\right)$ [42], and $T^{2} / Z_{4}$ $[43,44]$ orbifolds. Let us write down the action,

$$
S=\int d^{4} x \int_{-\pi R}^{\pi R} d y \int_{-\pi R}^{\pi R} d z\left[\mathcal{L}_{\text {bulk }}(x, y, z)+\sum_{\vec{y} \in \vec{y}_{i}} \delta\left(\vec{y}_{i}\right) \mathcal{L}_{\vec{y}_{i}}(x)\right]
$$

where $\vec{y}_{i}=\left(y_{i}, z_{i}\right)$ are orbifold fixed points. We note that terms localized at the fixed points are induced at quantum level even if we assume that they are vanishing at tree level $[7,52,53]$. For the orbifold we consider in this paper, the projections are

\footnotetext{
${ }^{5}$ In the $T^{2} / Z_{4}$ orbifold case, the condition $R_{y}=R_{z}$ is imposed by the consistency with the $Z_{4}$ discrete symmetry. See also [50] for a realization of $C P$ violation from the complex structure of $T^{2} / Z_{4}$, which appears in $4 \mathrm{D}$ effective interactions after $\mathrm{KK}$ decomposition.
} 
TABLE I. Fixed points which stem from each identification.

\begin{tabular}{lcc}
\hline \hline Type of orbifolding & Identification & Fixed points $\left(y_{i}, z_{i}\right)$ \\
\hline$T^{2} / Z_{2}$ & $(y, z) \sim(-y,-z)$ & $(0,0),(\pi R, 0),(0, \pi R),(\pi R, \pi R)$ \\
$T^{2} /\left(Z_{2} \times Z_{2}^{\prime}\right)$ & $(y, z) \sim(-y, z)$ and $(y, z) \sim(y,-z)$ & $(0,0),(\pi R, 0),(0, \pi R),(\pi R, \pi R)$ \\
$T^{2} / Z_{4}$ & $(y, z) \sim(-z, y)$ & $(0,0),(\pi R, \pi R)$ \\
\hline \hline
\end{tabular}

$$
\begin{gathered}
(y, z) \sim(-y,-z) \text { for } T^{2} / Z_{2}, \\
(y, z) \sim(-y, z) \text { and }(y, z) \sim(y,-z) \text { for } T^{2} /\left(Z_{2} \times Z_{2}^{\prime}\right), \\
(y, z) \sim(-z, y) \quad \text { for } T^{2} / Z_{4} .
\end{gathered}
$$

See also Table. I. In each case, we can choose a suitable boundary condition of 6D Weyl fermions, whose exact forms are not discussed in this paper, to generate 4D Weyl fermions at the zero modes.

The bulk Lagrangian, covariant derivatives, and field strengths of gauge bosons are essentially the same as that of the 5D mUED model except for the structure of spinors. For 6D Weyl fermions, the kinetic and Yukawa terms are

$$
\begin{aligned}
\mathcal{L}_{\text {kinetic }}= & -\overline{Q_{+}} \Gamma^{M} \mathcal{D}_{M} Q_{+}-\overline{U_{-}} \Gamma^{M} \mathcal{D}_{M} U_{-} \\
& -\overline{D_{-}} \Gamma^{M} \mathcal{D}_{M} D_{-}-\overline{L_{+}} \Gamma^{M} \mathcal{D}_{M} L_{+} \\
& -\overline{E_{-}} \Gamma^{M} \mathcal{D}_{M} E_{-}-\overline{N_{-}} \Gamma^{M} \mathcal{D}_{M} N_{-}, \\
\mathcal{L}_{\text {Yukawa }}= & -\lambda_{U} \overline{U_{-}}\left(Q_{+} \cdot \Phi\right)-\lambda_{D}\left(\overline{Q_{+}} \Phi\right) D_{-} \\
& -\lambda_{E}\left(\overline{L_{+}} \Phi\right) E_{-}+\text {H.c. },
\end{aligned}
$$

where the contraction of $S U(2)$ indices are understood. ${ }^{6}$ The resultant interactions relevant for our discussion are

$$
\begin{aligned}
\mathcal{L}_{\mathrm{KK} \text { top }}= & -i g_{4 s} \sum_{(m, n)}^{\infty}\left[\begin{array}{ll}
\bar{t}_{1} & \bar{t}_{2}
\end{array}\right]^{(m, n)} \gamma^{\mu} G_{\mu}^{(0)}\left[\begin{array}{l}
t_{1} \\
t_{2}
\end{array}\right]^{(m, n)} \\
& -\frac{m_{t}}{v_{\mathrm{EW}}} H^{(0)} \sum_{(m, n)}^{\infty}\left[\begin{array}{ll}
\bar{t}_{1} & \bar{t}_{2}
\end{array}\right]^{(m, n)} \\
& \times\left[\begin{array}{cc}
\sin 2 \alpha^{(m, n)} & -\gamma^{5} \cos 2 \alpha^{(m, n)} \\
\gamma^{5} \cos 2 \alpha^{(m, n)} & \sin 2 \alpha^{(m, n)}
\end{array}\right]\left[\begin{array}{l}
t_{1} \\
t_{2}
\end{array}\right]^{(m, n)},
\end{aligned}
$$

where $g_{4 s}=g_{s} /(2 \pi R)$ is the dimensionless $4 \mathrm{D} S U(3)_{C}$ coupling constant and $v_{\mathrm{EW}} \simeq 246 \mathrm{GeV}$ is the $4 \mathrm{D}$ Higgs vacuum expectation value, $G^{(0)}\left(H^{(0)}\right)$ shows zero-mode gluon (zero-mode physical Higgs), and $t_{1}^{(m, n)}, t_{2}^{(m, n)}$ are

TABLE II. The range of the parameter $(m, n)$ except the zeromode case $(m, n)=(0,0)$ in each case of the orbifolding.

\begin{tabular}{lc}
\hline \hline Type of orbifolding & Range of $(m, n)$ \\
\hline$T^{2} / Z_{2}$ & $m+n \geq 1$, or $m=-n \geq 1$ \\
$T^{2} /\left(Z_{2} \times Z_{2}^{\prime}\right)$ & $0 \leq m<\infty, 0 \leq n<\infty ;(m, n) \neq(0,0)$ \\
$T^{2} / Z_{4}$ & $1 \leq m<\infty, 0 \leq n<\infty$ \\
\hline \hline
\end{tabular}

mass eigenstates of the $(m, n)$ th KK-top quarks. Again, we only consider the KK top-quark loops since contributions from other flavors are suppressed by the small Yukawa coupling. Each mixing angle $\alpha^{(m, n)}$ is determined to be $\cos 2 \alpha^{(m, n)}=m_{(m, n)} / \sqrt{m_{t}^{2}+m_{(m, n)}^{2}}, \quad \sin 2 \alpha^{(m, n)}=$ $m_{t} / \sqrt{m_{t}^{2}+m_{(m, n)}^{2}}$. Each KK state is twofold degenerate and the $(m, n)$ th KK-top mass is

$$
m_{t,(m, n)}=\sqrt{m_{t}^{2}+m_{(m, n)}^{2}},
$$

with

$$
m_{(m, n)}:=\frac{\sqrt{m^{2}+n^{2}}}{R} .
$$

It should be mentioned that the difference from the mUED case appears only in the form of the KK mass and the number of d.o.f. in each KK level when we consider the gluon fusion process. We adopt $m(n)$ as the $y(z)$-directional KK index, whose parameter region is determined by the way of the orbifolding. This information has a great influence on the enhancement of the Higgs production through the gluon fusion.

\section{Real projective plane $\left(R P^{2}\right)$}

We can construct a UED model on a nonorientable geometry: real projective plane $\left(R P^{2}\right)$ [45]. $R P^{2}$ is defined by two types of identifications: a $\pi$ rotation $r$ and a glide $g$ :

$$
r:(y, z) \sim(-y,-z), \quad g:(y, z) \sim(y+\pi R,-z+\pi R) .
$$

The system is invariant under each manipulation in Eq. (20). Note that the shifts, $y \sim y+2 \pi R$ and $z \sim z+$ $2 \pi R$, can be obtained as different combinations of $r$ and $g$, respectively. Note also that no fixed point exists globally in this background geometry.

Under $r$ and $g$, Weyl fermions transforms as

$$
\begin{aligned}
& r: \Psi_{ \pm}(x ;-y,-z)=p_{r} \Gamma_{r} \Psi_{ \pm}(x ; y, z), \quad \Gamma_{r}=i \Gamma^{5} \Gamma^{6} \Gamma^{7}, \quad \text { (21) } \\
& g: \tilde{\Psi}_{ \pm}(x ; y+\pi R,-z+\pi R)=p_{g} \Gamma_{g} \Psi_{\mp}(x ; y, z), \quad \Gamma_{g}=\Gamma^{6} \Gamma^{7},
\end{aligned}
$$

where $\Gamma^{7}$ is the $6 \mathrm{D}$ chirality operator and $p_{r}, p_{g}\left(Z_{2}\right.$ parities) can take the value \pm 1 . The $\tilde{\Psi}_{ \pm}$is what we call the "mirror" fermion. Equation (21) has the same form as that of the $T^{2} / Z_{2}$ orbifold condition for $6 \mathrm{D}$ fermion. An

\footnotetext{
${ }^{6} \mathrm{We}$ leave the neutrino sector untouched since it is irrelevant for the Higgs signal considered in this paper.
} 
essential point of this model is that the condition (22) does not generate a 4D Weyl fermion in the zero-mode sector. In other words, the 6D chirality of both sides of Eq. (22) are different from each other. This means that we have to introduce new fermions $\tilde{\Psi}_{ \pm}$which have opposite 6D chirality and the same SM quantum number compared to each corresponding field $\Psi_{\mp}$. Concretely, mirror fermions,

$$
\mathcal{Q}_{-}, \mathcal{U}_{+}, \mathcal{D}_{+} ; \mathcal{L}_{-}, \mathcal{E}_{+}, \mathcal{N}_{+},
$$

are identified with $\left\{Q_{+}, U_{-}, D_{-} ; L_{+}, E_{-}, N_{-}\right\}$, respectively. The choice of $6 \mathrm{D}$ chiralities in Eq. (23) obeys the condition for realizing the $6 \mathrm{D}$ anomaly cancellation which we have argued before.

The bulk Lagrangian is the same as that of the $T^{2}$-based models using an orbifold, except for the existence of the mirror fermions:

$$
\begin{aligned}
\mathcal{L}_{\text {kinetic }}= & \frac{1}{2}\left[-\overline{Q_{+}} \Gamma^{M} \mathcal{D}_{M} Q_{+}-\overline{U_{-}} \Gamma^{M} \mathcal{D}_{M} U_{-}-\overline{D_{-}} \Gamma^{M} \mathcal{D}_{M} D_{-}\right. \\
& -\overline{L_{+}} \Gamma^{M} \mathcal{D}_{M} L_{+}-\overline{E_{-}} \Gamma^{M} \mathcal{D}_{M} E_{-}-\overline{N_{-}} \Gamma^{M} \mathcal{D}_{M} N_{-} \\
& -\overline{\mathcal{Q}_{-}} \Gamma^{M} \mathcal{D}_{M} \mathcal{Q}_{-}-\overline{\mathcal{U}_{+}} \Gamma^{M} \mathcal{D}_{M} \mathcal{U}_{+} \\
& -\overline{\mathcal{D}_{+}} \Gamma^{M} \mathcal{D}_{M} \mathcal{D}_{+}-\overline{\mathcal{L}_{-}} \Gamma^{M} \mathcal{D}_{M} \mathcal{L}_{-} \\
& \left.-\overline{\mathcal{E}_{+}} \Gamma^{M} \mathcal{D}_{M} \mathcal{E}_{+}-\overline{\mathcal{N}_{+}} \Gamma^{M} \mathcal{D}_{M} \mathcal{N}_{+}\right] \\
\mathcal{L}_{\text {Yukawa }}= & \frac{1}{2}\left[-\lambda_{U} \overline{U_{-}}\left(Q_{+} \cdot \Phi\right)-\lambda_{D}\left(\overline{Q_{+}} \Phi\right) D_{-}\right. \\
& -\lambda_{E}\left(\overline{L_{+}} \Phi\right) E_{-}-\lambda_{U} \overline{\mathcal{U}_{+}}\left(\mathcal{Q}_{-} \cdot \Phi\right) \\
& \left.-\lambda_{D}\left(\overline{\mathcal{Q}_{-}} \Phi\right) \mathcal{D}_{+}-\lambda_{E}\left(\overline{\mathcal{L}_{-}} \Phi\right) \mathcal{E}_{+}+\text {H.c. }\right]
\end{aligned}
$$

where we introduce the $1 / 2$ factors for later convenience. The neutrino sector is again left untouched as it is irrelevant for our discussion. By the use of Eq. (22), we can erase all the mirror fermions and obtain the ordinary form of the Lagrangian that is the same as the $T^{2}$ cases. The form of the $\pi$ rotation $r$ given in Eq. (21) is the same as that of the $Z_{2}$ orbifolding in Eq. (12). Therefore, the interactions of the $R P^{2}$ model, needed to calculate the gluon fusion process, take the same form as that of the $T^{2} / Z_{2}$ one given in Eq. (17).

\section{6D UED models based on $S^{2}$}

Let us review UED models based on the $S^{2}$ compactification. We span the extra dimension by the zenith and azimuthal angles $\theta$ and $\phi$, respectively. The two-sphere $S^{2}$ has a positive curvature and to stabilize the radius $R$, we introduce an extra $U(1)_{X}$ gauge field which has a monopole-like classical configuration [54],

$\left[\chi_{\phi}^{c}\left(x^{\mu}, \theta, \phi\right)\right]_{s}^{N}=\frac{n}{2 g_{X}}(\cos \theta \mp 1), \quad($ other components $)=0$,

where the superscript $c$ denotes the classical configuration, $g_{X}$ is the $6 \mathrm{D} U(1)_{X}$ gauge coupling, the integer $n$ is the (negative) monopole charge, and the superscripts $N$ and
$S$ indicate that the field is given in north (involving the $\theta=0$ point) and south (involving the $\theta=\pi$ point) charts, respectively. The $U(1)_{X}$ transition function from the north to the south chart is given by

$\left[\chi_{M}\left(x^{\mu}, \theta, \phi\right)\right]^{S}=\left[\chi_{M}\left(x^{\mu}, \theta, \phi\right)\right]^{N}+\frac{1}{g_{X}} \partial_{M} \alpha\left(x^{\mu}, \theta, \phi\right)$,

with $\alpha\left(x^{\mu}, \theta, \phi\right)=n \phi$. Because of the monopole-like configuration, the radius of $S^{2}$ is stabilized spontaneously at

$$
R^{2}=\left(\frac{n}{2 g_{X} M_{*}^{2}}\right)^{2}
$$

where $M_{*}$ is the $6 \mathrm{D}$ Planck scale.

We mention that any 6D field $\Xi$ on $S^{2}$ is KK expanded by use of the spin-weighted spherical harmonics ${ }_{s} Y_{j m}(\theta, \phi)$ as follows:

$$
\begin{aligned}
\Xi(x, \theta, \phi)^{N} & =\sum_{j=|s|}^{\infty} \sum_{m=-j}^{j} \xi^{(j, m)}(x) f_{\Xi}^{(j, m)}(\theta, \phi)^{N}, \\
f_{\Xi}^{(j, m)}(\theta, \phi)^{N} & :=\frac{{ }_{s} Y_{j m}(\theta, \phi) e^{ \pm i s \phi}}{R},
\end{aligned}
$$

where $\xi^{(j, m)}$ is the $(j, m)$ th expanded 4D field, $f_{\Xi}^{(j, m)}$ is the corresponding mode function, and $s$ is the spin weight of the field $\Xi$. The spin-weighted spherical harmonics ${ }_{s} Y_{j m}(\theta, \phi)$ match the orthonormal condition as

$\int_{0}^{2 \pi} d \phi \int_{-1}^{1} d \cos {\overline{\theta_{s}}}_{Y_{j m}(\theta, \phi)}^{s} Y_{j^{\prime} m^{\prime}}(\theta, \phi)=\delta_{j j^{\prime}} \delta_{m m^{\prime}}$

A spin weight of a fermion is closely related to its $U(1)_{X}$ charge. When we assign $U(1)_{X}$ charges of 6D Weyl fermions $\Psi_{ \pm}$as $q_{\Psi_{ \pm}}$, the corresponding spin weights of 4D Weyl fermions $\left\{\bar{\psi}_{+_{R}^{L}}^{L}, \psi_{-}^{L}\right\}$ are given as follows in our convention:

$$
s_{+_{R}^{L}}=\frac{n q_{\Psi_{+}} \pm 1}{2}, \quad s_{-_{R}^{L}}=\frac{n q_{\Psi_{-}} \mp 1}{2} .
$$

Note that if a 6D Weyl fermion takes a spin weight $s=0$, a $j=0$ mode appears as a 4D Weyl fermion with vanishing KK mass. This means that we can get chiral SM fermions without orbifolding in the case of $S^{2}$. When we take the values,

$$
\left(s_{+R}, s_{+L}, s_{-R}, s_{-L}\right)=(0,1,1,0),
$$

we can create the same situation as in the $T^{2}$-based models discussed before. The spin weight of the 4D-vector component of a $6 \mathrm{D}$ gauge boson is always $s=0$ and then there is a zero-mode that can be identified as the SM gauge boson. On the other hand, extra dimensional components of the $6 \mathrm{D}$ gauge boson are expanded by the $|s|=1$ spin-weighted spherical harmonics and have no zero mode.

In our configuration, any $(j, m)$ th KK mode has the KK mass:

$$
m_{(j, m)}=\frac{\sqrt{j(j+1)}}{R} .
$$


An important point is that the form of the above KK mass is independent of the index of $m$. This means that there are $2 j+1$ degenerate modes for each $j$. Note that the lightest KK mode has the mass $\sqrt{2} / R$.

As discussed above, the $4 \mathrm{D}$-vector component of a $6 \mathrm{D}$ gauge boson has a zero mode. This is the case for the extra $U(1)_{X}$ gauge boson too. Phenomenologically, the existence of an extra $U(1)$ interaction, under which SM fields are charged, is problematic [46]. In the following, let us see how to get rid of this massless $U(1)_{X}$ vector.

\section{Projective sphere}

We can construct a UED model compactified on the PS, a sphere $S^{2}$ with its antipodal points being identified by $(\theta, \phi) \sim(\pi-\theta, \phi+\pi)$ [46]. In the UED model based on the PS, the $6 \mathrm{D}$ action takes a different form from that of the 6D orbifold UED models. One of the remarkable points of this model is that there is no fixed point on the background geometry PS. As in the $R P^{2}$ model, we introduce mirror $6 \mathrm{D}$ Weyl fermions:

$$
\mathcal{Q}_{-}, \mathcal{U}_{+}, \mathcal{D}_{+} ; \mathcal{L}_{-}, \mathcal{E}_{+}, \mathcal{N}_{+},
$$

which have opposite 6D chirality and opposite SM and $U(1)_{X}$ charges, compared to the fields $\left\{Q_{+}, U_{-}, D_{-}\right.$; $\left.L_{+}, E_{-}, N_{-}\right\}$. Because of the existence of mirror fermions the kinetic term takes the same form as in the $R P^{2}$ model (24) and the Yukawa interaction is modified to

$$
\begin{aligned}
\mathcal{L}_{\text {Yukawa }}= & \frac{1}{2}\left[-\lambda_{U} \overline{U_{-}}\left(Q_{+} \cdot \Phi\right)-\lambda_{D}\left(\overline{Q_{+}} \Phi\right) D_{-}\right. \\
& -\lambda_{E}\left(\overline{L_{+}} \Phi\right) E_{-}-\lambda_{U}^{*} \overline{\mathcal{U}_{+}}\left(\mathcal{Q}_{-} \cdot \Phi\right) \\
& \left.-\lambda_{D}^{*}\left(\overline{\mathcal{Q}_{-}} \Phi\right) \mathcal{D}_{+}-\lambda_{E}^{*}\left(\overline{\mathcal{L}_{-}} \Phi\right) \mathcal{E}_{+}+\text {H.c. }\right] .
\end{aligned}
$$

Like the $R P^{2}$ case, which we have discussed before, we introduce the $1 / 2$ factors for a later convenience. The covariant derivatives in this model are given as

$$
\left.\mathcal{D}_{M}=\partial_{M}+i g_{s} G_{M}^{a} T_{s}^{a}+i g \mathcal{W}_{M}^{a} T^{a}+i g_{Y} \mathcal{B}_{M} Y \text { (for } \Phi\right) \text {, }
$$

$$
\begin{aligned}
\mathcal{D}_{M}= & \partial_{M}+i g_{s} G_{M}^{a} T_{s}^{a}+i g \mathcal{W}_{M}^{a} T^{a}+i g_{Y} \mathcal{B}_{M} Y \\
& +i g_{X} q_{\Psi}\left(\mathcal{X}_{M}^{c}+\chi_{M}\right)+\Omega_{M} \\
& \left(\text { for } Q_{+}, U_{-}, D_{-} ; L_{+}, E_{-}, N_{-}\right), \\
\mathcal{D}_{M}= & \partial_{M}+i g_{s} G_{M}^{a}\left[-T_{s}^{a}\right]^{\mathrm{T}}+i g \mathcal{W}_{M}^{a}\left[-T^{a}\right]^{\mathrm{T}} \\
& +i g_{Y} \mathcal{B}_{M}[-Y]+i g_{X} q_{\Psi}\left(\mathcal{X}_{M}^{c}+\chi_{M}\right)+\Omega_{M} \\
& \left(\text { for } \mathcal{Q}_{-}, \mathcal{U}_{+}, \mathcal{D}_{+} ; \mathcal{L}_{-}, \mathcal{E}_{+}, \mathcal{N}_{+}\right),
\end{aligned}
$$

where $\Omega_{M}$ is the spin connection. The covariant derivative of Higgs is the same as that in the $S^{2} / Z_{2}$ case, but there is a difference between those of fermions and these mirror fermions. We discuss these points shortly below.

As we mentioned before, the projective sphere is a nonorientable manifold and has no fixed point. Let us consider the 6D $P$ and $C P$ transformations. Under the antipodal projection,

$$
\begin{aligned}
\mathcal{X}_{\mu}(x, \pi-\theta, \phi+\pi)^{N} & =\chi_{\mu}^{C}(x, \theta, \phi)^{S}, \\
\chi_{\theta}(x, \pi-\theta, \phi+\pi)^{s} & =-\chi_{\theta}^{C}(x, \theta, \phi)^{S}, \\
\left\{\chi_{\phi}^{c}, \chi_{\phi}\right\}(x, \pi-\theta, \phi+\pi)^{s} & =\left\{\left(\chi_{\phi}^{c}\right)^{C}, \chi_{\phi}^{C}\right\}(x, \theta, \phi)^{S},
\end{aligned}
$$

where the superscript $C$ denotes the 6D $C$ transformation. Recall that the superscript $c$ denotes the classical configuration. These conditions leave the monopole-like configuration invariant under the antipodal identification and projects out the unwanted $U(1)_{X} 4 \mathrm{D}$-vector zero mode. In contrast, the identification of a SM gauge boson $\mathcal{A}_{M}^{(i)}$ should be done by another condition since we want the corresponding 4D-vector zero mode, where $i$ shows the type of gauge group. We adopt the 6D $P$ transformation and those identifications are written as

$$
\begin{aligned}
& \mathcal{A}_{\mu}^{(i)}(x, \pi-\theta, \phi+\pi)^{N}=\mathcal{A}_{\mu}^{(i)}(x, \theta, \phi)^{S}, \\
& \mathcal{A}_{\theta}^{(i)}(x, \pi-\theta, \phi+\pi)^{N}=-\mathcal{A}_{\theta}^{(i)}(x, \theta, \phi)^{S}, \\
& \mathcal{A}_{\phi}^{(i)}(x, \pi-\theta, \phi+\pi)^{N}=\mathcal{A}_{\phi}^{(i)}(x, \theta, \phi)^{S},
\end{aligned}
$$

where it is evident that the zero mode of $\mathcal{A}_{\mu}^{(i)}$ survives. We also identify Higgs with the 6D $P$ transformation to obtain its zero mode:

$$
\Phi(x, \pi-\theta, \phi+\pi)^{N}=\Phi(x, \theta, \phi)^{S} .
$$

Finally, we discuss the identification of 6D Weyl fermions. Since 6D Weyl fermions have a $U(1)_{X}$ charge and interact with the $U(1)_{X}$ gauge boson, they should be identified by the $6 \mathrm{D} C P$ transformation. The specific form of the $6 \mathrm{D} C P$ transformation, for example, in the case of $U_{-}$, is as follows:

$$
\mathcal{U}_{+}(x, \pi-\theta, \phi+\pi)^{N}=P U_{-}^{C}(x, \theta, \phi)^{S},
$$

where the matter field $U_{-}$is identified to the mirror $\mathcal{U}_{+}$. We decide the forms of covariant derivatives (37) and (38) on the criterion of invariance of the action under the 6D CP transformation. Using the identification conditions (39)-(42), we can see that the mirror fermions drop out of the action after the identifications and eventually we obtain the usual type of UED model action. This can be interpreted such that all the modes of mirror fermions $\left\{\mathcal{Q}_{-}, \mathcal{U}_{+}, \mathcal{D}_{+} ; \mathcal{L}_{-}, \mathcal{E}_{+}, \mathcal{N}_{+}\right\}$are erased and no mode of $\left\{Q_{+}, U_{-}, D_{-} ; L_{+}, E_{-}, N_{-}\right\}$is projected out. The interaction terms which we need for calculation in this model is the same as those in Eq. (17). The only difference is the number of degenerate top KK modes in each $j$ level.

\section{2. $S^{2} U E D$ with a Stueckelberg field $\left(S^{2}\right)$}

As a solution to the massless $U(1)_{X}$ problem, we can simply give a Stueckelberg mass $[55,56]$ (see also $[57,58]$ for reviews) to the $U(1)_{X}$ field. We can make the unwanted 
$U(1)_{X} 4 \mathrm{D}$-vector zero mode to be massive while preserving the classical monopole structure in Eq. (26). This way, we can formulate a UED model on $S^{2}$ with no field identification. Let us call this simple model the $S^{2}$ UED model. In the $S^{2}$ UED model, the matter contents, bulk Lagrangian, definition of field strengths, and covariant derivatives and the configuration of the classical $U(1)_{X}$ field are the same as the PS model after removing the mirror fermions, except for the Stueckelberg field part. In contrast to the $S^{2} / Z_{2}$ orbifold below, there is neither a fixed point nor a localized Lagrangian anywhere on $S^{2}$, as in the case of PS UED. The d.o.f. of KK fermions has no difference between the $S^{2}$ UED and PS one (after the antipodal projection). There is no need for an additional computation; all we have to do is to borrow the PS result as a whole when we are only interested in the gluon fusion process.

\section{3. $S^{2} / Z_{2}$ orbifold}

Although the above $S^{2}$ UED model with a Stueckelberg $U(1)_{X}$ mass is already phenomenologically viable, we may further perform a $Z_{2}$ orbifolding on it [47]. ${ }^{7}$ On this orbifold, the point $(\theta, \phi)$ is identified with $(\pi-\theta,-\phi)$. The 6D action $S$ is as follows:

$$
\begin{aligned}
S= & \int d^{4} x \int_{0}^{\pi} d \theta \int_{0}^{2 \pi} d \phi \sqrt{-g}\left[\mathcal{L}_{\text {bulk }}(x, y, z)+\delta\left(\theta-\frac{\pi}{2}\right)\right. \\
& \left.\times \delta(\phi) \mathcal{L}_{(\pi / 2,0)}(x)+\delta\left(\theta-\frac{\pi}{2}\right) \delta(\phi-\pi) \mathcal{L}_{(\pi / 2, \pi)}(x)\right]
\end{aligned}
$$

where $\sqrt{-g}=R^{2} \sin \theta$. This system has two fixed points of the $Z_{2}$ symmetry at $(\theta, \phi)=\left(\frac{\pi}{2}, 0\right),\left(\frac{\pi}{2}, \pi\right)$ and we describe the localized terms with $\mathcal{L}_{(\pi / 2,0)}, \mathcal{L}_{(\pi / 2, \pi)}$, respectively. Like the $T^{2}$ case, we do not discuss those parts in this paper.

We can easily construct mode functions of $S^{2} / Z_{2}$ $f_{s, t}^{(j, m)}(\theta, \phi)$ with spin weight $s$ in both north and south charts following the general prescription [59] as follows:

$$
f_{s, t}^{(j, m)}(\theta, \phi)^{N}= \begin{cases}\frac{1}{2 R}\left[{ }_{s} Y_{j m}(\theta, \phi)+(-1)^{j-s}{ }_{s} Y_{j-m}(\theta, \phi)\right] e^{ \pm i s \phi} & \text { for } t=+1 \\ \frac{1}{2 R}\left[{ }_{s} Y_{j m}(\theta, \phi)-(-1)^{j-s}{ }_{s} Y_{j-m}(\theta, \phi)\right] e^{ \pm i s \phi} & \text { for } t=-1,\end{cases}
$$

where $t= \pm 1$ is the $Z_{2}$ parity. These mode functions have the property that $f_{s, t= \pm 1}^{(j, m)}(\pi-\theta,-\phi)^{N}= \pm f_{s, t= \pm 1}^{(j, m)}(\theta, \phi)^{S}$. To realize the $Z_{2}$ symmetry, we identify a field at $(\theta, \phi)$ in the north chart with the same field at $(\pi-\theta,-\phi)$ in the south chart.

The range of the summation over $m$ shrinks from $[-j, j]$ to $[0, j]$ after the $Z_{2}$ identification. Under the transformation of $(\theta, \phi) \rightarrow(\theta, \phi+\pi)$, mode functions behave as

$$
\begin{gathered}
f_{s=0, t=+1}^{(j, m)}(\theta, \phi+\pi)^{N}=(-1)^{m} f_{s=0, t=+1}^{(j, m)}(\theta, \phi)^{N}, \\
f_{s= \pm 1, t=-1}^{(j, m)}(\theta, \phi+\pi)^{N}=-(-1)^{m} f_{s= \pm 1, t=-1}^{(j, m)}(\theta, \phi)^{N} .
\end{gathered}
$$

After some fields redefinition, we can find that each KK field has a KK parity $(-1)^{m}$, which is a remnant of the KK angular momentum conservation.

We focus on the $m=0$ modes of each $j$ level. When we see the concrete forms of mode functions in $m=0$, which are

$$
\begin{gathered}
f_{s=0, t=+1}^{(j, m=0)}(\theta, \phi)^{N}=\frac{1}{2 R}\left(1+(-1)^{j}\right)_{0} Y_{j 0}(\theta, \phi), \\
f_{s=+1, t=-1}^{(j, m=0)}(\theta, \phi)^{N}=\frac{1}{2 R}\left(1+(-1)^{j}\right)_{1} Y_{j 0}(\theta, \phi) e^{ \pm i \phi},
\end{gathered}
$$

\footnotetext{
${ }^{7}$ This extra $Z_{2}$ cannot project out the $U(1)_{X}$ gauge field. In [47], the $U(1)_{X}$ is assumed to be broken by an anomaly. Since we need a classical configuration of the $U(1)_{X}$, it would be theoretically preferable to break it by a tiny Stueckelberg mass.
}

$f_{s=-1, t=-1}^{(j, m=0)}(\theta, \phi)^{N}=\frac{1}{2 R}\left(1+(-1)^{j}\right)_{-1} Y_{j 0}(\theta, \phi) e^{\mp i \phi}$,

we find that $m=0$ modes appear only in the case of even $j$. Then the degeneracy of KK masses is

$$
j+1 \text { for } j \text { : even, } j \text { for } j \text { : odd, }
$$

since $m$ runs from 0 to $j$. Again, this mode counting is the only important point when computing the enhancement of the Higgs production via gluon fusion process. We do not discuss the form of interactions which we need for calculating the gluon fusion process because there is essentially no difference from the $T^{2}$ case (17).

\section{HIGGS PRODUCTION AND DECAY INTO FOUR LEPTONS IN UED MODELS}

In the SM, the cross section for the leading order (LO) one-loop Higgs production via the gluon fusion process and its subsequent decay into a $Z$ boson pair: $g g \rightarrow H \rightarrow$ $Z Z$ is given by [60]. The LO parton-level cross section shown in Fig. 1 is

$$
\begin{aligned}
\hat{\sigma}_{g g \rightarrow H \rightarrow Z Z}^{\mathrm{SM}}= & \frac{\alpha_{4 s}^{2}}{256 \pi^{3}}\left(\frac{m_{Z}}{v_{\mathrm{EW}}}\right)^{4}\left[1+\frac{\left(\hat{s}-2 m_{Z}^{2}\right)^{2}}{8 m_{Z}^{4}}\right] \\
& \times \frac{\hat{s}}{\left(\hat{s}-M_{H}^{2}\right)^{2}+\Delta^{2}} \sqrt{1-\frac{4 m_{Z}^{2}}{\hat{s}}}|I(\hat{s})|^{2},
\end{aligned}
$$

where $m_{W}, m_{Z}, m_{t}$, and $M_{H}$ are, respectively, the $W, Z$, top quark, and Higgs boson masses, $\alpha_{4 s}=g_{4 s}^{2} / 4 \pi$ is the $4 D$ 


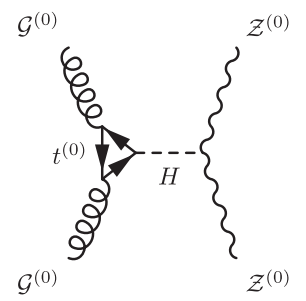

FIG. 1. Feynman diagram which describes the dominant contribution to the gluon fusion Higgs production process and the subsequent decay to $2 Z$. $G^{(0)}$ and $Z^{(0)}$ are gluon and $Z$ boson zero modes, respectively.

QCD gauge coupling, $\hat{s}$ is the center-of-mass-energysquared of the scattering partons; we employ the normalization for the Higgs vacuum expectation value: $v_{\mathrm{EW}}^{2}=$ $1 / \sqrt{2} G_{F} \simeq(246 \mathrm{GeV})^{2}$, and the loop functions are defined as

$$
\begin{gathered}
I(\lambda)=-2 \lambda+\lambda(1-4 \lambda) \int_{0}^{1} \frac{d x}{x} \ln \left[\frac{x(x-1)}{\lambda}+1-i \epsilon\right] \\
\tilde{I}(\lambda)=\lambda \int_{0}^{1} \frac{d x}{x} \ln \left[\frac{x(x-1)}{\lambda}+1-i \epsilon\right]
\end{gathered}
$$

The explicit result of the integral is

$$
\begin{aligned}
& \int_{0}^{1} \frac{d x}{x} \ln \left[\frac{x(x-1)}{\lambda}+1-i \epsilon\right] \\
& = \begin{cases}-2\left[\arcsin \frac{1}{\sqrt{4 \lambda}}\right]^{2} & \left(\text { for } \lambda \geq \frac{1}{4}\right) \\
\frac{1}{2}\left[\ln \frac{1+\sqrt{1-4 \lambda}}{1-\sqrt{1-4 \lambda}}-i \pi\right]^{2} & \left(\text { for } \lambda<\frac{1}{4}\right) .\end{cases}
\end{aligned}
$$

We have also defined $\tilde{I}$ for later use for the Dirichlet Higgs model. In Eq. (50), we have taken into account the total decay width of the Higgs in its propagator:

$$
\Delta=M_{H} \Gamma_{H} .
$$

In the current analysis, we take into account the Higgs decay into $W, Z$ and top-quark pairs, which are dominant when we consider the heavy SM Higgs boson: $M_{H} \geq 2 m_{W}$. The explicit form is shown in Eq. (C27) in Appendix C. Note that we take into account only the top-quark loop in the SM cross section (50), given by the diagram shown in Fig. 1, since the Yukawa coupling to others are negligible compared to the top one. We have also ignored the contributions from the subleading box diagrams [61]. See Appendix C for further discussion on how to take into account the width.

\section{A. Gluon fusion process in UED models}

In this paper, we consider the KK-top-loop contributions to the gluon fusion process in several UED models, namely, the 5D UED model on $S^{1} / Z_{2}$ (mUED) [1], DH [40], 6D UED model on $T^{2} / Z_{2}$ [1], $T^{2} / Z_{4}$ [43,44], $T^{2} /\left(Z_{2} \times Z_{2}^{\prime}\right)$ [42], real projective plane $\left(R P^{2}\right)$ [45],
$S^{2}=Z_{2}$ [47], PS [46], and $S^{2}$ with a Stueckelberg field $\left(S^{2}\right)$. We have given a brief review on these models in the previous section. The contribution from KK-top loops to the gluon fusion process is analogous to that of the top loop in the SM and the difference resides only in the loop function. The relevant Feynman diagram is shown in Figs. 2 and 3. The effective vertex, which is represented by the lined blob in the diagram, includes the contributions to the gluon fusion from the zero-mode top quark and the KK-top quarks. Since the zero-mode sector of the UED model regenerates the SM configuration, the result of the former contribution is the same as that of the SM in Eq. (50). The forms of the latter contribution will be shown later. We note that the letter $H$ in Figs. 2 and 3 shows the zero-mode physical Higgs boson in all the cases except for the DH model where $H$ stands for the first KK Higgs.

For each model, we get the following result, where $J_{\text {model }}$ indicates the corresponding loop function:

$$
\begin{aligned}
\hat{\sigma}_{g g \rightarrow H \rightarrow Z Z}^{\text {model }}= & \frac{\alpha_{4 s}^{2}}{256 \pi^{3}}\left(\frac{m_{Z}}{v_{\mathrm{EW}}}\right)^{4} \frac{1+\left(\hat{s}-2 m_{Z}^{2}\right)^{2}}{8 m_{Z}^{4}} \\
& \times \frac{\hat{s}}{\left(\hat{s}-M_{H}^{2}\right)^{2}+\left(M_{H} \Gamma_{H}\right)^{2}} \\
& \times \sqrt{1-\frac{4 m_{Z}^{2}}{\hat{s}}} K\left|J_{\text {model }}(\hat{s})\right|^{2},
\end{aligned}
$$

with

$$
J_{\mathrm{mUED}}(\hat{s})=I\left(\frac{m_{t}^{2}}{\hat{s}}\right)+2 \sum_{n=1}^{\infty}\left(\frac{m_{t}}{m_{t(n)}}\right)^{2} I\left(\frac{m_{t(n)}^{2}}{\hat{s}}\right),
$$



FIG. 2. A schematic description of the dominant contribution to the gluon fusion Higgs production process and the subsequent decay to $2 Z$. The lined blob indicates the effective vertex.

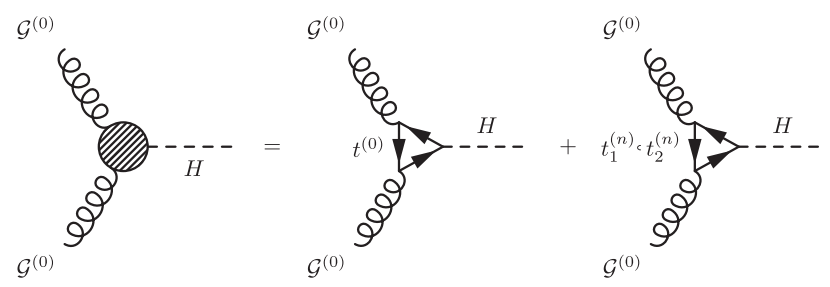

FIG. 3. The effective vertex which describes the Higgs production from the gluon fusion. 


$$
\begin{aligned}
& \begin{array}{l}
J_{\mathrm{DH}}(\hat{s})=\sqrt{2} \varepsilon_{1} \sqrt{\mid I\left(\frac{m_{t}^{2}}{\hat{s}}\right)+2 \sum_{n=1}^{\infty}} \\
(\hat{s}) \\
\left.\frac{m_{t}^{2}}{\hat{s}}\right)+2 \sum_{\substack{m+n \geq 1 \\
\text { or } m=-n \geq 1}}\left(\frac{m_{t}}{m_{t(m, n)}}\right)^{2} I\left(\frac{m_{t(m, n)}^{2}}{\hat{s}}\right),
\end{array} \\
& J_{T^{2} / Z_{4}}(\hat{s})=I\left(\frac{m_{t}^{2}}{\hat{s}}\right)+2 \sum_{m \geq 1, n \geq 0}\left(\frac{m_{t}}{m_{t(m, n)}}\right)^{2} I\left(\frac{m_{t(m, n)}^{2}}{\hat{s}}\right), \\
& J_{T^{2} / Z_{2} \times Z_{2}^{\prime}}(\hat{s})=I\left(\frac{m_{t}^{2}}{\hat{s}}\right)+2 \sum_{\substack{m \geq 0, n \geq 0 \\
(m, n) \neq(0,0)}}\left(\frac{m_{t}}{m_{t(m, n)}}\right)^{2} I\left(\frac{m_{t(m, n)}^{2}}{\hat{s}}\right), \\
& J_{S^{2} / Z_{2}}(\hat{s})=I\left(\frac{m_{t}^{2}}{\hat{s}}\right)+2 \sum_{j=1}^{j_{\max }}\left(\frac{m_{t}}{m_{t(j)}}\right)^{2} n(j) I\left(\frac{m_{t(j)}^{2}}{\hat{s}}\right), \\
& J_{\mathrm{PS}}(\hat{s})=J_{S^{2}}(\hat{s})=I\left(\frac{m_{t}^{2}}{\hat{s}}\right)+2 \sum_{j=1}^{j_{\max }}\left(\frac{m_{t}}{m_{t(j)}}\right)^{2}(2 j+1) I\left(\frac{m_{t(j)}^{2}}{\hat{s}}\right),
\end{aligned}
$$

where the KK-top masses are given by

$$
\begin{gathered}
m_{t(n)}:=\sqrt{m_{t}^{2}+\frac{n^{2}}{R^{2}}}=\sqrt{m_{t}^{2}+n^{2} M_{\mathrm{KK}}^{2}}, \\
m_{t(m, n)}:=\sqrt{m_{t}^{2}+\frac{m^{2}+n^{2}}{R^{2}}}=\sqrt{m_{t}^{2}+\left(m^{2}+n^{2}\right) M_{\mathrm{KK}}^{2}}, \\
m_{t(j)}:=\sqrt{m_{t}^{2}+\frac{j(j+1)}{R^{2}}}=\sqrt{m_{t}^{2}+\frac{j(j+1) M_{\mathrm{KK}}^{2}}{2}} .
\end{gathered}
$$

Here the $M_{\mathrm{KK}}$ is the first KK mass, which is written as

$$
M_{\mathrm{KK}}=\frac{1}{R}
$$

for the compactifications based on $S^{1} / Z_{2}$, interval, and $T^{2}$ [namely, the mUED, DH, $T^{2} / Z_{2}, R P^{2}, T^{2} / Z_{4}$, and $T^{2} /\left(Z_{2} \times Z_{2}^{\prime}\right)$ models] and is written as

$$
M_{\mathrm{KK}}=\frac{\sqrt{2}}{R}
$$

for the $S^{2}$-based ones (namely, the $S^{2} / Z_{2}$, PS, and $S^{2}$ models). The gluon fusion process for mUED in $S^{1} / Z_{2}$ is first shown in Ref. [37] and for $S^{2} / Z_{2}$ in Ref. [38]. Also, it has been calculated for $T^{2} / Z_{4}$ and PS in Ref. [39]. The results for $\mathrm{DH}, T^{2} / Z_{2}, R P^{2}, T^{2} /\left(Z_{2} \times Z_{2}^{\prime}\right)$, and $S^{2}$ are newly presented in this paper. The factor $\sqrt{2} \varepsilon_{1}$ in Eq. (57) is equal to $2 \sqrt{2} / \pi \sim 0.9$. The origin of this suppression factor is nonorthonormality of mode functions on an interval. In the case of the $S^{2}$-based compactification, there are some degenerated states, the number of which is described with $n(j)$ on $S^{2} / Z_{2}$ and $(2 j+1)$ on PS or $S^{2}$ for each KK index $j$. The specific form of $n(j)$ for the orbifold $S^{2} / Z_{2}$ in Eq. (61) is as follows:

$$
n(j)= \begin{cases}j+1 & \text { for } j: \text { even } \\ j & \text { for } j: \text { odd }\end{cases}
$$

Several comments are in order.

(i) The origin of the factor 2 in front of each $\mathrm{KK}$ summation is the fact there are both left-and righthanded (namely, vectorlike) KK modes for each chiral quark zero mode.

(ii) All the KK contributions are positive and hence always enhance the Higgs production rate via the gluon fusion process, except for the DH model in which the zero-mode Higgs contribution is absent.

(iii) Each value of Yukawa couplings of KK quarks to the Higgs is the same as that of the coupling between the corresponding zero-mode fermion and the Higgs. We only consider triangle loop diagrams of the SM top quark and its KK excited modes because their Yukawa coupling to the Higgs is dominant compared to that of other fermions.

(iv) In each $\mathrm{KK}$ summation, infinite numbers of $\mathrm{KK}$ modes contribute to the process in principle. In $6 \mathrm{D}$, these summations are divergent and a suitable scheme of regularization is required. $j_{\max }$ in Eqs. (61) and (62) shows an upper bound of the summation over the index $j$. Further discussion will be shown in the following subsection.

(v) $K$ in Eq. (55) is the so-called $\mathrm{K}$ factor, a phenomenological approximation in order to naively take into account higher-order QCD corrections. One may take $K \sim 2$ for Tevatron and $K \sim 1.5-1.6$ for the LHC, respectively [32]. In the limit where the KK loop is viewed as a contribution to the effective Higgs-gluon-gluon coupling, the QCD corrections to Higgs production are very similar between the $\mathrm{SM}$ and the new physics contributions. The reason is that the Higgs-gluon-gluon coupling always has the same structure, and only its coefficient changes. This is discussed in detail, e.g., in Ref. [62] in the context of supersymmetry (but it works the same way in UED as in supersymmetry). Therefore, we have included a $\mathrm{K}$ factor also for the new physics terms as in Eq. (55).

(vi) When the compactification radius is too large, namely, when the first KK $W$ is lighter than half the Higgs mass, the Higgs can decay into a pair of KK particles and its decay width $\Gamma_{H}$ becomes 
broader. In this situation it becomes harder to find the evidence of the Higgs boson and hence we restrict ourselves within the region where the Higgs mass is smaller than twice the first KK $W$ mass so that such a decay mode does not open up.

(vii) Although the one-loop gluon fusion process is the dominant production channel of the Higgs, its contribution to the Higgs total decay width is smaller at least by 3 orders of magnitude compared to the decay into a $W$ pair in the case of the SM with $M_{H} \geq 300 \mathrm{GeV}$ [32]. Even after the enhancement of $\mathcal{O}(10)$ from KK-top contributions, the decay into the gluon pair is still negligible.

(viii) In Eq. (55), the Higgs decay width is taken into account by the naive Breit-Wignar formula in the denominator. When the Higgs mass is large, say $M_{H}=700 \mathrm{GeV}$, the Higgs decay width is as large as $180 \mathrm{GeV}$. In some literature, the expression $M_{H} \Gamma_{H}$ in the Breit-Wignar formula is replaced by $\hat{s} \Gamma_{H} / M_{H}$. In Appendix $\mathrm{C}$ we discuss reliability of our treatment.

\section{B. UV cutoff scale in six dimensions}

In 6D UED models, since the gluon fusion process is UV divergent, we must consider the upper limit of the summations of the KK number in such models. ${ }^{8}$ First let us briefly review how the naive dimensional analysis (NDA) is applied to the higher-dimensional theory. Following the concept of NDA, a loop expansion parameter $\epsilon$ in the $D$-dimensional $S U(N)$ gauge theory at a scale $\mu$ is obtained as

$$
\epsilon(\mu)=\frac{1}{2} \frac{2 \pi^{D / 2}}{(2 \pi)^{D} \Gamma(D / 2)} N_{g} g_{D i}^{2}(\mu) \Lambda^{D-4},
$$

where $N_{g}$ is a group index, $g_{D i}$ is a dimensionful gauge coupling in $D$ dimensions, and $\Lambda$ is a UV cutoff scale. The index $i$ is introduced to express the type of gauge interaction and the remaining part originates from the $D$-dimensional momentum loop integral. The cutoff scale $\Lambda$ is the scale where the perturbation breaks down $\epsilon(\Lambda) \sim 1$.

Precisely speaking, the dimensionful higher-dimensional gauge coupling does not "run." Let us explain what is meant by the running coupling in (69), basically following Ref. [48]. When we consider a 6D theory $(D=6)$ with two compact spacial dimensions, an effective 4D gauge coupling $g_{4 i}$ emerges after KK decomposition: $g_{4 i}=g_{6 i} / \sqrt{V_{2}}$, where $V_{2}$ is the volume of two extra dimensions. Concretely, $V_{2}=(2 \pi R)^{2}$ and $4 \pi R^{2}$ for $T^{2}$ and $S^{2}$, respectively. In this paper, we employ a bottom-up approach for the running gauge coupling. At energies below the first $\mathrm{KK}$ scale, the theory is purely four dimensional (after integrating out all the massive modes of order KK scale)

\footnotetext{
${ }^{8}$ In the 5 D UED model, we can execute this mode summation with no divergence.
}

and the gauge coupling runs logarithmically. Let us then increase the energy scale. Every time we cross a KK mass scale, there open up the corresponding KK modes to run in the loops in the gauge boson two-point function. In all the scales, the theory is renormalizable and the running of the gauge coupling is logarithmic. However, due to the increase of the number of particles in the loops, the running of the gauge coupling becomes effectively a power law at the energy scales much above the first KK scale. This way, we get the effective power-law running of the gauge coupling, within a purely renormalizable approach. In this paper, we neglect possible threshold corrections at the UV cutoff scale, since we are interested in what is the highest possible $\Lambda$ that can be consistent with the low energy theory.

In the above stated strategy, we get the following running of the 4D effective gauge coupling strength $\alpha_{4 i}(\mu)$,

$$
\begin{aligned}
\alpha_{4 i}^{-1}(\mu) \simeq & \alpha_{4 i}^{-1}\left(m_{Z}\right)-\frac{\mathrm{b}_{i}^{\mathrm{SM}}}{2 \pi} \ln \frac{\mu}{m_{Z}}+2 C \frac{\mathrm{b}_{i}^{6 \mathrm{D}}}{2 \pi} \ln \frac{\mu}{M_{\mathrm{KK}}} \\
& -C \frac{\mathrm{b}_{i}^{6 \mathrm{D}}}{2 \pi}\left[\left(\frac{\mu}{M_{\mathrm{KK}}}\right)^{2}-1\right],
\end{aligned}
$$

where $C=\pi / 2$ and 1 for $T^{2}$ and $S^{2}$, respectively. In Eq. (70), we have approximated that all the masses are degenerate in each KK level. Depending on the models, some fraction of the KK modes are projected out, but we assume that all of them contribute to the running, in order to give the most conservative upper bound on the UV cutoff scale. A more detailed explanation is given in Appendix B.

While the described procedure gives a reasonable estimate of the UV cutoff scale, one has to be aware that this is not much more than an order-of-magnitude estimate. We will plot our results for the maximum and minimum values of the UV cutoff scale that are theoretically reasonable. In Table III, we list our choice of bounds at which the KK mode summation is truncated to regularize the process.

\section{Convolution of parton distribution}

Let us briefly review the standard prescription to estimate the event number of the Higgs production in $p p \rightarrow$ $Z Z \rightarrow 4 \ell$ (four leptons) via the gluon fusion process as a function of the invariant mass of $Z Z$, given the parton-level cross section, where $4 \ell$ denotes two pairs consisting of either $e^{-} e^{+}$or $\mu^{-} \mu^{+}$, since a tau pair is less visible at the LHC. That is, the final state is possibly $e^{-} e^{+} e^{-} e^{+}$, $\mu^{-} \mu^{+} \mu^{-} \mu^{+}$, or $e^{-} e^{+} \mu^{-} \mu^{+}$. Because of the large mass difference between the $Z$ boson and two electrons or two muons, the subsequent processes, $Z \rightarrow e^{+} e^{-}$and $Z \rightarrow$ $\mu^{+} \mu^{-}$are well treated with on-shell approximation.

By using the parton distribution function of gluon $f_{g}(x, \hat{s})$, we give the formula of the total cross section of $p p \rightarrow Z Z:$

$$
\sigma_{p p \rightarrow Z Z}^{\text {model }}(s)=\int_{0}^{1} d \tau \hat{\sigma}_{g g \rightarrow H \rightarrow Z Z}^{\text {model }}(\tau s) L(\tau, s),
$$


TABLE III. Our choices of maximum and minimum upper bounds for KK indices and for the corresponding UV cutoff scale.

\begin{tabular}{lcccc}
\hline \hline & \multicolumn{2}{c}{$T^{2}$-based } & \multicolumn{2}{c}{$S^{2}$-based } \\
\hline & $\max$ & $\min$ & $\max$ & $\min$ \\
\hline KK-index & $m^{2}+n^{2} \leq 28$ & $m^{2}+n^{2} \leq 10$ & $j(j+1) \leq 90$ & $j(j+1) \leq 30$ \\
UV cutoff & $\Lambda_{6 D} \sim 5 M_{\mathrm{KK}}$ & $\Lambda_{6 D} \sim 3 M_{\mathrm{KK}}$ & $\Lambda_{6 D} \sim 7 M_{\mathrm{KK}}$ & $\Lambda_{6 D} \sim 4 M_{\mathrm{KK}}$ \\
\hline \hline
\end{tabular}

TABLE IV. The region of KK summation for the maximum UV cutoff.

\begin{tabular}{lc}
\hline \hline Geometry & Allowed region of KK indices \\
\hline$T^{2} / Z_{2}$ or $R P^{2}$ & $(m, n)=(1,0),(2,0),(3,0),(4,0),(5,0),(0,1),(1,1),(2,1),(3,1),(4,1),(5,1),(-1,2),(0,2),(1,2),(2,2)$, \\
& $(3,2),(4,2),(-2,3),(-1,3),(0,3),(1,3),(2,3),(3,3),(4,3),(-2,3),(-1,3),(0,3),(1,3),(2,3)$, \\
& $(3,3),(4,3),(-3,4),(-2,4),(-1,4),(0,4),(1,4),(2,4),(3,4),(-1,5),(0,5),(1,5),(1,-1),(2,-1)$, \\
$T^{2} /\left(Z_{2} \times Z_{2}^{\prime}\right)$ & $(3,-1),(4,-1),(5,-1),(2,-2),(3,-2),(4,-2),(3,-3),(4,-3)$ \\
$T^{2} / Z_{4}$ & $(m, n)=(1,0),(2,0),(3,0),(4,0),(5,0),(0,1),(1,1),(2,1),(3,1),(4,1),(5,1),(0,2),(1,2),(2,2)$, \\
& $(3,2),(4,2),(0,3),(1,3),(2,3),(3,3),(4,3),(0,4),(1,4),(2,4),(3,4),(0,5),(1,5)$ \\
$S^{2} / Z_{2}$ & $(m, n)=(1,0),(2,0),(3,0),(4,0),(5,0),(1,1),(2,1),(3,1),(4,1),(5,1),(1,2),(2,2)$, \\
PS or $S^{2}$ & $(3,2),(4,2),(1,3),(2,3),(3,3),(4,3),(1,4),(2,4),(3,4),(1,5)$ \\
\hline \hline & $j=1-9[n(j)=j+1$ for $j:$ even or $n(j)=j$ for $j:$ odd $]$ \\
$j=1-9(2 j+1$ degenerated states $)$
\end{tabular}

where we define

$$
L(\tau, s):=\int_{-\ln (1 / \sqrt{\tau})}^{\ln (1 / \sqrt{\tau})} d y f_{g}\left(\sqrt{\tau} e^{y}, \tau s\right) f_{g}\left(\sqrt{\tau} e^{-y}, \tau s\right)
$$

The concrete form of $\hat{\sigma}_{p p \rightarrow Z Z}^{\text {model }}$ has been shown in Eqs. (55)-(62). The invariant mass of $Z Z$ is represented as $M_{Z Z}^{2}=\hat{s}=\tau s$. Then, the differential cross section of $p p \rightarrow Z Z \rightarrow 4 \ell$ as a function of $M_{Z Z}$ is written as

$$
\begin{aligned}
\frac{d \sigma_{p p \rightarrow Z Z \rightarrow 4 \ell}^{\text {model }}\left(M_{Z Z}\right)}{d M_{Z Z}}= & \frac{2 M_{Z Z}}{s} \times \hat{\sigma}_{g g \rightarrow H \rightarrow Z Z}^{\text {model }}\left(M_{Z Z}^{2}\right) \\
& \times L\left(\frac{M_{Z Z}^{2}}{s}, s\right) \times 4 \mathrm{Br}(Z \rightarrow 2 \ell)^{2},
\end{aligned}
$$

where $\operatorname{Br}(Z \rightarrow 2 \ell):=\operatorname{Br}\left(Z \rightarrow e^{+} e^{-}\right)=\operatorname{Br}\left(Z \rightarrow \mu^{+} \mu^{-}\right)=$ 0.034 is the branching ratio.

\section{NUMERICAL RESULTS}

We use the top-quark mass $m_{t}=172 \mathrm{GeV}$ and the LO QCD running coupling. We apply the CTEQ5 LO parton distribution function of gluon [63]. In our analysis, we consider only KK scales large enough not to open up the Higgs decay channel into a pair of KK-top, $Z$, and $W$ particles. When the decay channel into two KK particles opens up, the Higgs resonance tends to become too broad and hard to be seen at Tevatron and LHC. We list in Table IV the KK modes that satisfy the maximum cutoff criterion given in Table III.

\section{A. Tevatron}

We evaluate the $H \rightarrow Z Z \rightarrow 4 \ell$ event number for each $25 \mathrm{GeV}$ bin of $M_{Z Z}$, expected at Tevatron with an integrated luminosity $8 \mathrm{fb}^{-1}$ at $\sqrt{s}=1.96 \mathrm{TeV}$ in the above mentioned UED models. We show the results for the Higgs mass $M_{H}=330 \mathrm{GeV}$. We consider the KK scales $M_{\mathrm{KK}}=200,400$, and $800 \mathrm{GeV}$, except for the DH model which does not have a zero-mode Higgs. For the DH model, we take the first KK Higgs as the Higgs field, that is, $M_{\mathrm{KK}}=M_{H}=330 \mathrm{GeV} .^{9}$ The results are shown in Fig. 4. We see that the event number is enhanced in all the UED models from the SM one. In particular, the $6 \mathrm{D}$ projective sphere model can give a large enhancement in the Higgs production by a factor as large as 100 compared to the SM when the KK scale is low at $200 \mathrm{GeV} .{ }^{10}$ Two $Z Z \rightarrow 4 \ell$ events are observed in a $300-350 \mathrm{GeV}$ bin at the Collider Detecter at Fermilab with the integrated luminosity $4.8 \mathrm{fb}^{-1}$ [64] and two such events are observed in $325-375 \mathrm{GeV}$ bins at D0 with $6.4 \mathrm{fb}^{-1}$ [65]. Recently, two more events are reported around $330 \mathrm{GeV}$ [66]. However, the above large cross section to explain the Tevatron data is found to be inconsistent with the LHC data $[67,68]$.

\footnotetext{
${ }^{9}$ Note that the coupling of the Dirichlet Higgs to the SM modes is decreased by a factor 0.9 compared to the SM Higgs. ${ }^{10}$ Even if the one-loop $H \rightarrow g g$ decay rate is enhanced by the factor 100 from the SM one, it is still subdominant compared with the tree level $H \rightarrow W W$ [32] and we neglect its contribution to the total decay width of the Higgs.
} 

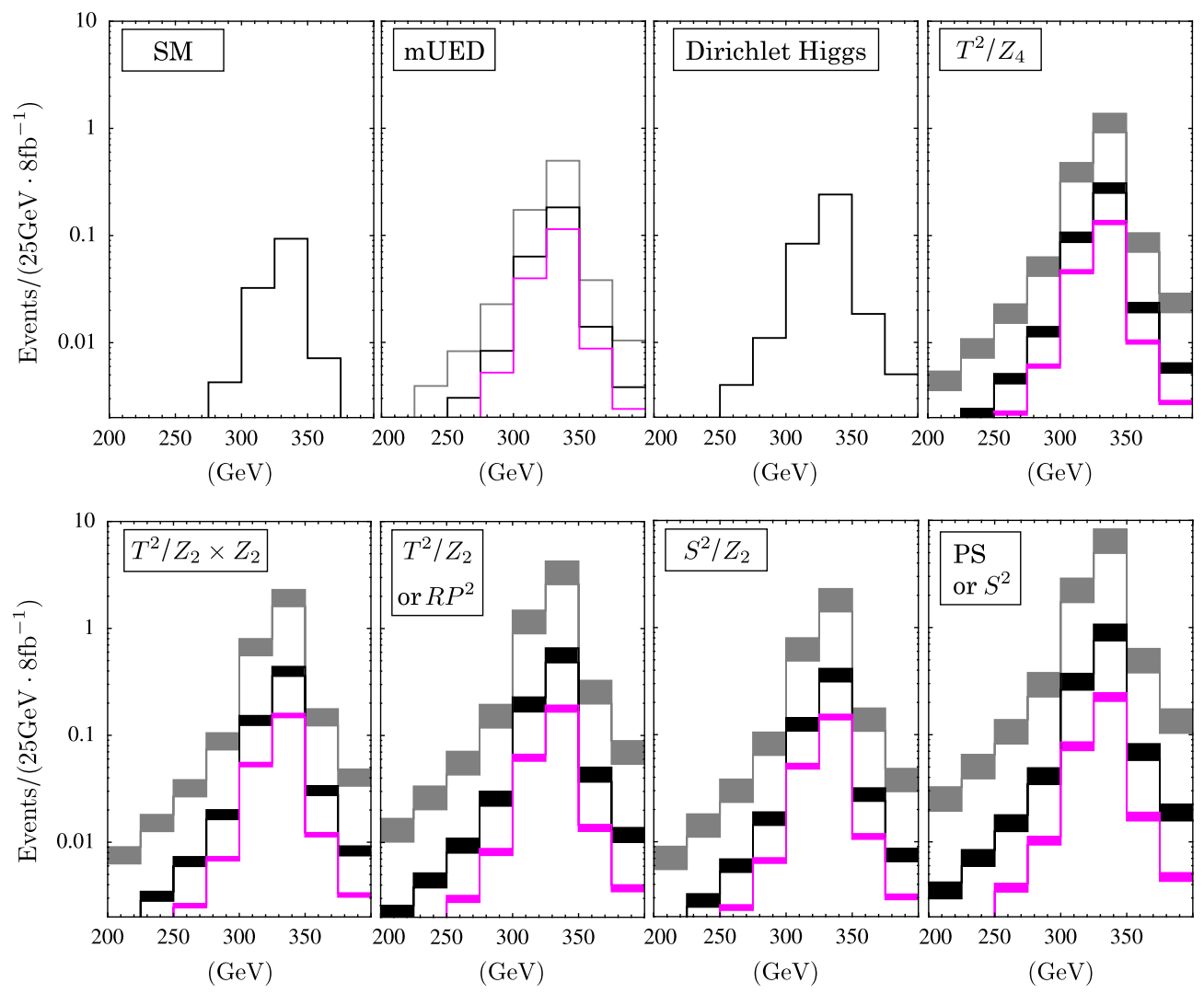

FIG. 4 (color online). The $H \rightarrow Z Z \rightarrow 4 \ell$ event number for each $25 \mathrm{GeV}$ bin of $M_{Z Z}$ for $M_{H}=330 \mathrm{GeV}$, expected at Tevatron with an integrated luminosity $8 \mathrm{fb}^{-1}$ at $\sqrt{s}=1.96 \mathrm{TeV}$. The grey (uppermost), black (middle), and magenta (lowermost) lines represent the expected event number with $M_{\mathrm{KK}}=200,400$, and $800 \mathrm{GeV}$, respectively. For 6D UED models, we consider dependency on the UV cutoff, whose range is from minimum (lower side of band) to maximum (upper side of band) given in Table III.

\section{B. LHC}

We plot the event number of $H \rightarrow Z Z \rightarrow 4 \ell$ for the Higgs mass $M_{H}=500 \mathrm{GeV}$ at the LHC with an integrated luminosity $10 \mathrm{fb}^{-1}$ at $\sqrt{s}=7 \mathrm{TeV}$ in Fig. 5. When $\sqrt{s}=$ $7 \mathrm{TeV}$, we have checked that we cannot see a sizable number of events for all the UED models with $M_{H}=$ $700 \mathrm{GeV}$ even for an integrated luminosity $10 \mathrm{fb}^{-1}$, which is expected by the end of 2012 after which the LHC is planned to be (shut down for a year and then) upgraded to 13-14 TeV. Therefore, we show corresponding results for $M_{H}=700 \mathrm{GeV}$ at $\sqrt{s}=14 \mathrm{TeV}$ with an integrated luminosity $10 \mathrm{fb}^{-1}$ in Fig. 6. We have chosen several KK scales: 400, 800, $1200 \mathrm{GeV}$. We show our plots in logarithmic scale so that one can easily see the results for different luminosities by simply shifting them upward or downward.

In Fig. 5, with an integrated luminosity $10 \mathrm{fb}^{-1}$, we can see in total a few events in 5D UED models (mUED and $\mathrm{DH})$ and $\mathcal{O}(1)-\mathcal{O}(10)$ events for each $25 \mathrm{GeV}$ bin for 6D UED models. Note that even if we can only see at best in total a few events for 5D UED by the end of 2012, this $Z Z \rightarrow 4 \ell$ channel is virtually background free at $500 \mathrm{GeV}$ and the result would be still significant.

In Fig. 6, for $M_{H}=700 \mathrm{GeV}$, we have plotted the results for the upgraded energy $\sqrt{s}=14 \mathrm{TeV}$. We see that even with the integrated luminosity $10 \mathrm{fb}^{-1}, 6 \mathrm{D}$ UED models can have a few events for each $25 \mathrm{GeV}$ bin if the KK scale is relatively low $M_{\mathrm{KK}}=400 \mathrm{GeV}$. The 6D $T^{2} / Z_{2}, R P^{2}, S^{2}$, and PS models can have in total few events for the higher $\mathrm{KK}$ mass $M_{\mathrm{KK}}=800 \mathrm{GeV}$. When the integrated luminosity adds up to $100 \mathrm{fb}^{-1}$, we can see a few events for each bin for 5D mUED and DH models, and even for the SM (though the SM itself cannot satisfy the electroweak constraints on the $S, T$ parameters, contrary to the UED models). We see that the Dirichlet Higgs model has a slightly smaller cross section than the standard model. This is because the KK scale is fixed to be large, $700 \mathrm{GeV}$, and hence the enhancement of the KK top loop is small, while the Yukawa coupling of the (first KK) Higgs to the top quark is decreased by the factor $2 \sqrt{2} / \pi \simeq 0.9$.

Let us emphasize that the enhancement of Higgs production $g g \rightarrow H$ in UED does not depend on the details of the model such as the mass structure at the orbifold fixed points. Parameter dependence is only on the Higgs mass and the KK scale. In this sense, this Higgs channel signal is complementary to the direct search of the KK modes decaying into LKP [14,28], which is nice because of the directness but is dependent on the details of the KK mass splitting from the boundary terms. 

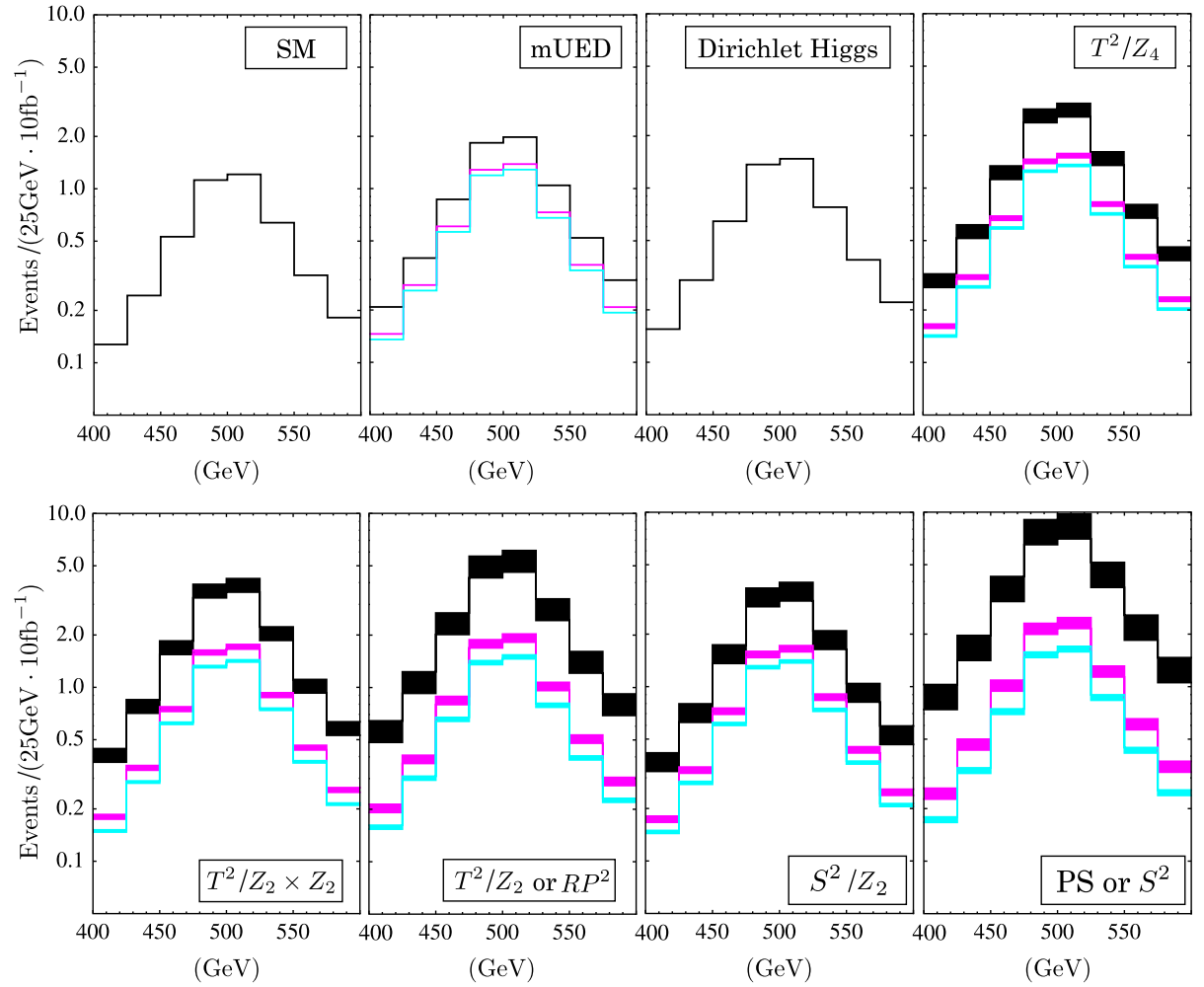

FIG. 5 (color online). The $H \rightarrow Z Z \rightarrow 4 \ell$ event number for each $25 \mathrm{GeV}$ bin of $M_{Z Z}$ for $M_{H}=500 \mathrm{GeV}$, expected at the LHC with an integrated luminosity $10 \mathrm{fb}^{-1}$ at $\sqrt{s}=7 \mathrm{TeV}$. The black (uppermost), magenta (middle), and cyan (lowermost) lines represent the expected event number with $M_{\mathrm{KK}}=400,800$, and $1200 \mathrm{GeV}$, respectively. Dependence on the 6D UV cutoff scale is shown to be the same as in Fig. 4 .
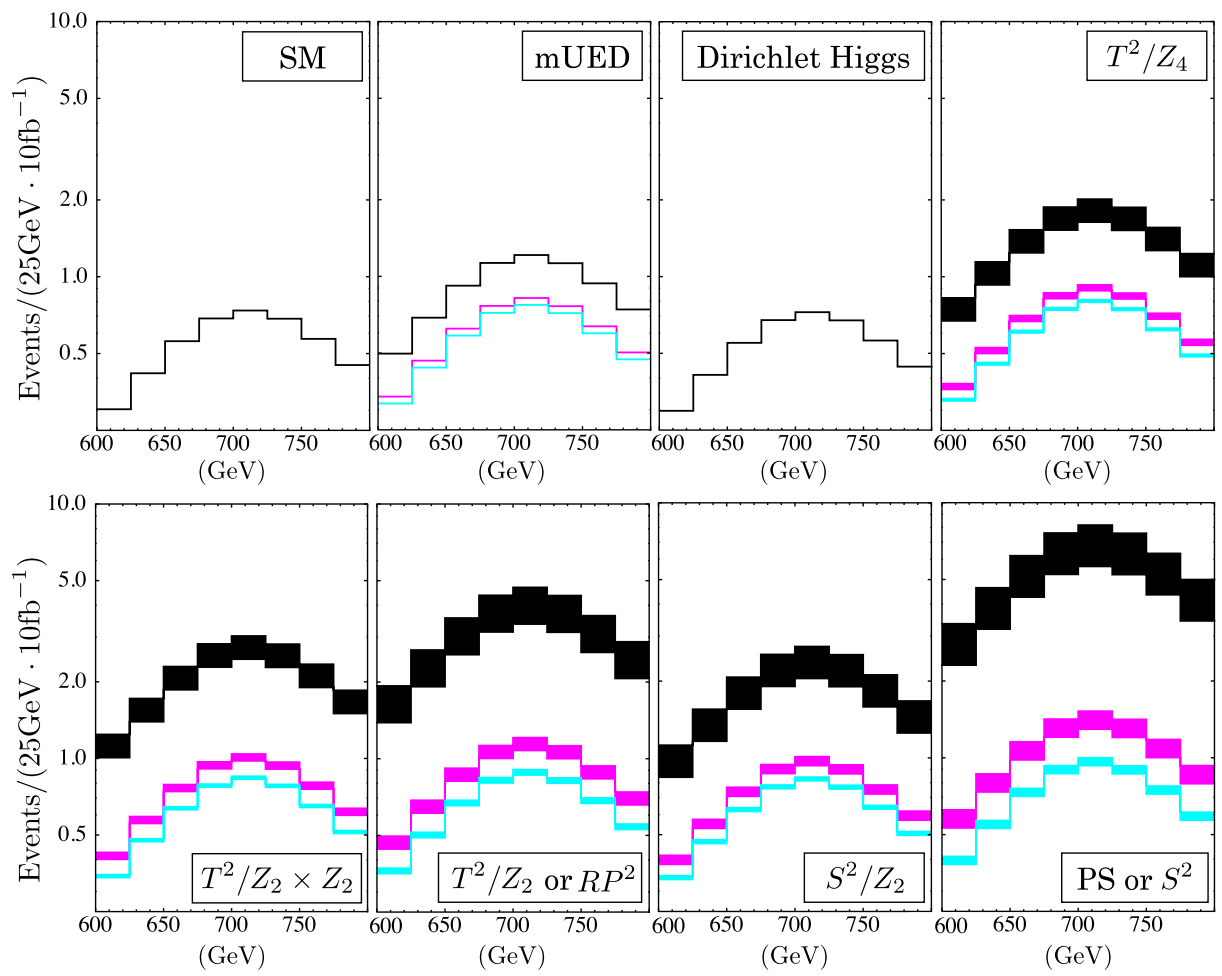

FIG. 6 (color online). The $H \rightarrow Z Z \rightarrow 4 \ell$ event number for each $25 \mathrm{GeV}$ bin of $M_{Z Z}$ for $M_{H}=700 \mathrm{GeV}$, expected at LHC with an integrated luminosity $10 \mathrm{fb}^{-1}$ at $\sqrt{s}=14 \mathrm{TeV}$. The black (uppermost), magenta (middle), and cyan (lowermost) lines represent the expected event number with $M_{\mathrm{KK}}=400,800$, and $1200 \mathrm{GeV}$, respectively. Dependence on the 6D UV cutoff scale is shown to be the same as in Fig. 4 . 


\section{SUMMARY AND DISCUSSIONS}

We have presented a review on the known $5 \mathrm{D}$ and $6 \mathrm{D}$ UED models focusing on the relevant part to the gluon fusion process. We have explained our computation of the gluon fusion process including the KK-top-quark loops, which is new for the $T^{2} / Z_{2}, T^{2} /\left(Z_{2} \times Z_{2}^{\prime}\right), R P^{2}$, and $S^{2}$ UED models. For 6D UED models, we have shown an NDA analysis of the highest possible UV cutoff scale in the $S^{2}$-based compactification, extending the analysis of Refs. [3,48] on the $T^{2}$ compactification.

For Higgs mass, $M_{H}=500 \mathrm{GeV}$, we can see a few (virtually background free) $H \rightarrow Z Z \rightarrow 4 \ell$ events in $5 \mathrm{D}$ UED models with $10 \mathrm{fb}^{-1}$ of integrated luminosity. The 6D UED models can further exhibit the shape of the resonance if the KK scale is relatively low. When the Higgs mass is as large as $M_{H}=700 \mathrm{GeV}$, we found no parameter region that can be seen within the integrated luminosity of $\mathcal{O}(10) \mathrm{fb}^{-1}$ at $\sqrt{s}=7 \mathrm{TeV}$. We have also studied the event rate for the upgraded energy $\sqrt{s}=14 \mathrm{TeV}$ when $M_{H}=700 \mathrm{GeV}$. We see that the 5D and 6D UED models typically require $100 \mathrm{fb}^{-1}$ and $10 \mathrm{fb}^{-1}$ of data, respectively, in order to establish the existence of the resonance. As is reported in Ref. [39], the 6D UED model on the projective sphere (or $S^{2}$ ) shows the greatest enhancement of the Higgs production via gluon fusion process, among all the known UED models.

Let us again emphasize that the presented Higgs signal of UED needs only the Higgs mass and KK scale as input parameters, is independent of the detailed KK mass splitting, and hence is unaffected by the boundary or fixedpoint mass structures. Therefore, this Higgs signal of UED is complementary to the direct KK resonance production and to the dark matter signal. As the enhancement of the Higgs production via the gluon fusion process can be so large, recent data from the LHC $[67,68]$ can already significantly exclude the parameter space of the UED models. This analysis is presented in a separate publication [69]. We will also show a combined bound from the triviality and the electroweak precision constraints in addition to that of Ref. [69]. (The triviality bound would lower the maximum allowed UV cutoff scale when the Higgs mass is heavy.) In this paper, we have studied the cleanest possible signature $H \rightarrow Z Z \rightarrow 4 \ell$. It is expected that a combined analysis, including other decay channels such as $H \rightarrow W W$ and $H \rightarrow Z Z \rightarrow \ell \ell \nu \nu$, will provide a large gain over all individual analyses [68]. Such a combined analysis for the UED models will be presented elsewhere.

\section{ACKNOWLEDGMENTS}

We are grateful to Shoji Asai for valuable comments and thank Abdelhak Djouadi, Kaoru Hagiwara, Kazunori Hanagaki, Shigeki Matsumoto, Kazuki Sakurai, and
Kohsaku Tobioka for helpful communications. We appreciate the referee's thorough and constructive comments. The work of K. O. is partially supported by Scientific Grants from Ministry of Education and Science (Japan), No. 20244028, No. 23104009, and No. 23740192.

\section{APPENDIX A: FEYNMAN RULES FOR DIRICHLET HIGGS MODEL}

In this appendix, we describe the Feynman rules that are necessary for our computation. The mass terms of KK fermions are

$$
-\sum_{n=1}^{\infty}\left[\begin{array}{ll}
\bar{Q}_{t} & \bar{U}_{t}
\end{array}\right]^{(n)}\left[\begin{array}{cc}
\frac{n}{R} & m_{t} \\
m_{t} & -\frac{n}{R}
\end{array}\right]\left[\begin{array}{l}
Q_{t} \\
U_{t}
\end{array}\right]^{(n)},
$$

where $Q_{t}$ is an upper component of the quark doublet in third generation and $U_{t}$ is the top-quark singlet. Transforming each KK states by the following unitary transformation including chiral rotation:

$$
\left[\begin{array}{l}
t_{1} \\
t_{2}
\end{array}\right]{ }^{(n)}=\left[\begin{array}{ll}
\gamma^{5} & \\
& 1
\end{array}\right]\left[\begin{array}{cc}
\cos \alpha^{(n)} & -\sin \alpha^{(n)} \\
\sin \alpha^{(n)} & \cos \alpha^{(n)}
\end{array}\right]\left[\begin{array}{l}
Q_{t} \\
U_{t}
\end{array}\right]^{(n)},
$$

we can obtain the ordinary diagonalized Dirac mass terms, where $t_{1}^{(n)}$ and $t_{2}^{(n)}$ are mass eigenstates of $n$th KK-top quarks and each mixing angle $\alpha^{(n)}$ is determined to be $\cos 2 \alpha^{(n)}=$ $(n / R) / \sqrt{m_{t}^{2}+n^{2} / R^{2}}, \sin 2 \alpha^{(n)}=m_{t} / \sqrt{m_{t}^{2}+n^{2} / R^{2}}$. Each KK state is twofold degenerate and $n$th KK-top mass is $m_{t,(n)}=\sqrt{m_{t}^{2}+n^{2} / R^{2}}$. The corresponding interaction terms are

$$
\begin{aligned}
\mathcal{L}_{\mathrm{KK} \text { top }}= & -i g_{4 s} \sum_{n=1}^{\infty}\left[\begin{array}{ll}
\bar{t}_{1} & \bar{t}_{2}
\end{array}\right]^{(n)} \gamma^{\mu} \mathcal{G}_{\mu}^{(0)}\left[\begin{array}{l}
t_{1} \\
t_{2}
\end{array}\right]^{(n)} \\
& -\frac{m_{t}}{v_{\mathrm{EW}}} \sum_{n, m=1}^{\infty}\left[\begin{array}{ll}
\bar{t}_{1} & \bar{t}_{2}
\end{array}\right]^{(n)} H^{(m)} \\
& \times\left(\sqrt{2} \varepsilon_{m}+\frac{1}{\sqrt{2}}\left(\varepsilon_{2 n+m}-\varepsilon_{2 n-m}\right) \gamma^{5}\right) \\
& \times\left[\begin{array}{cc}
\sin 2 \alpha^{(n)} & -\gamma^{5} \cos 2 \alpha^{(n)} \\
\gamma^{5} \cos 2 \alpha^{(n)} & \sin 2 \alpha^{(n)}
\end{array}\right]\left[\begin{array}{l}
t_{1} \\
t_{2}
\end{array}\right]^{(n)},
\end{aligned}
$$

where $g_{4 s}$ is a dimensionless $4 \mathrm{D} S U(3)_{C}$ coupling constant and $v_{\mathrm{EW}}$ is the 4D Higgs vacuum expectation value which appears after the KK expansion. $G_{\mu}^{(0)}$ is a massless gluon and $H^{(m)}$ is $m$ th KK Higgs bosons. The concrete shape of the factor of $\sqrt{2} \varepsilon_{n}$ is $2 \sqrt{2} / n \pi$, whose origin is the nonorthonormality of mode functions in the Dirichlet Higgs model. In the Dirichlet Higgs model there is no zero-mode Higgs because of choosing Dirichlet boundary condition in Higgs field. The first KK Higgs boson behaves like a heavy SM Higgs except that its interaction with the SM fields are multiplied by $\sqrt{2} \varepsilon_{1}$. The explicit form of the Feynman rules is 


$$
\begin{aligned}
& \underset{H^{(m)} ; p}{-----}=\frac{i}{-p^{2}-(m / R)^{2}+i \epsilon}
\end{aligned}
$$

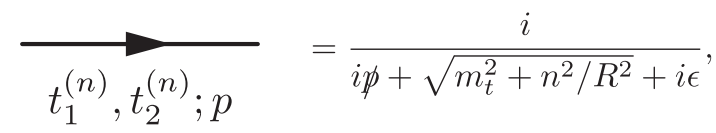

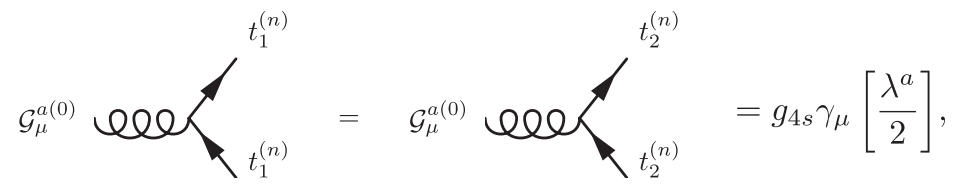

$$
\begin{aligned}
& H^{(m)}-\cdots-\left\langle H_{t_{1}^{(n)}}^{t_{1}^{(n)}}=--H_{t_{2}^{(n)}}^{t_{2}^{(n)}}=-i \frac{m_{t}}{v_{\mathrm{EW}}}\left(\sqrt{2} \varepsilon_{m}+\frac{1}{\sqrt{2}}\left(\varepsilon_{2 n+m}-\varepsilon_{2 n-m}\right) \gamma^{5}\right) \sin 2 \alpha^{(n)}\right.
\end{aligned}
$$

where $a$ is a gluon (adjoint) color index and $\lambda^{a}$ are the Gell-Mann matrices. It is noted that we can find an interaction which is proportional to the $\gamma^{5}$ matrix at the $\mathrm{KK}$ Higgs Yukawa couplings to KK-top quarks, which generates another type of contribution to the Higgs production through the gluon fusion process. ${ }^{11}$ This type of contribution does not exist in ordinary 5D or 6D UED models.

\section{APPENDIX B: UV CUTOFF BASED ON RGE ANALYSIS IN KK PICTURE}

In this appendix, we show the details of the renormalization group analysis, based on the strategy stated in Sec. III B that follows Ref. [48]. We note that we do not need any regularization of the infinite $\mathrm{KK}$ sum, employed in Ref. [48], in our bottom-up approach. A higher-dimensional gauge theory is equivalent to the corresponding 4D theory with an infinite tower of KK modes. It suggests that all we have to do for deriving a running effect of the 4D effective gauge coupling at leading order is to count the d.o.f. of fields whose masses are lower compared to a reference energy $\mu$. This important information is encoded into the coefficient $\mathrm{b}_{i}$. The formula for the running coupling of 4D $S U(N)$ or $U(1)$ gauge theory is well known as follows:

$$
\begin{aligned}
& \mathrm{b}_{S U(N)}=\left(-\frac{d 1}{3} \sum_{4 \mathrm{D} \text { vectors }}^{\frac{d}{d \ln \mu} \alpha_{4 i}^{-1}=-\frac{1}{2 \pi} \mathrm{b}_{i},}\right. \\
& \left.+\frac{1}{3} \sum_{4 \mathrm{D} \text { Higgs }} C(r)+\frac{1}{6} \sum_{\substack{4 \mathrm{D} \text { adjiont } \\
\text { scalars }}} C_{2} \text { (Adjoint) }\right),
\end{aligned}
$$

\footnotetext{
${ }^{11}$ This situation is similar to the famous fact that $\pi^{0} \rightarrow 2 \gamma$ decay is enhanced through a chiral anomaly [70].
}

$$
\mathrm{b}_{U(1)}=\left(\frac{2}{3} \sum_{\substack{4 \mathrm{D} \text { Weyl } \\ \text { fermions }}} Y_{f}^{2}+\frac{1}{3} \sum_{4 \mathrm{D} \text { Higgs }} Y_{H}^{2}\right)
$$

where $\alpha_{4 i}$ is a 4D gauge coupling strength, whose group is discerned by $i ; C(r)$ is defined by $\operatorname{tr}\left[T_{r}^{a} T_{r}^{b}\right]=C(r) \delta^{a b}$ for each representation of the $S U(N)$ group and the specific value is $1 / 2$ for the fundamental representation. $C_{2}$ (Adjoint) is the quadratic Casimir operator for the adjoint representation, whose value is $N ; Y_{f}\left(Y_{H}\right)$ shows the $U(1)$ charge of the 4D Weyl fermion (Higgs). The contribution from $\mathrm{KK}$ vector bosons is included in the first term in Eq. (B2). We note that a 6D gauge field has two extradimensional components and that a $4 \mathrm{D}$ adjoint scalar is left as a physical mode after the $\mathrm{KK}$ decomposition. [When counting the number of adjoint scalar degrees of freedom in the running (B1), it is two rather than one in our treatment.]

We can obtain the solution of Eq. (B1) by the integration of the both sides over the region $\left[m_{Z}, \mu\right]$. The essential point is that the coefficient $b_{i}$ becomes altered when the reference energy $\mu$ crosses a threshold and the number of the effectively massless d.o.f. of fields under the $\mu$ changes. Neglecting the KK mass splitting from the electroweak symmetry breaking, the threshold correction from $l$ th KK particles arises simultaneously when $\mu$ exceeds the value of the $l$ th KK mass. This approximation simplifies the calculation to estimate the effect from $l$ th KK particles all at once. The values of $\mathrm{b}_{i}$ in the zero modes $\left(\mathrm{b}_{i}^{\mathrm{SM}}\right)$ and $l$ th KK modes $\left(b_{i}^{6 \mathrm{D}}\right)$ from the bulk SM matter contents are summarized in Table V. ${ }^{12}$

\footnotetext{
${ }^{12}$ Note that we do not employ the grand unified theory normalization for the $U(1)_{Y}$ coupling and the beta function.
} 
TABLE V. RGE coefficients in Eq. (C3).

Gauge group $\mathrm{SM}$ contribution $\left(\mathrm{b}_{i}^{\mathrm{SM}}\right) \quad \mathrm{KK}$ contribution $\left(\mathrm{b}_{i}^{6 \mathrm{D}}\right)$

\begin{tabular}{lcc}
\hline$S U(3)_{C}$ & -7 & -2 \\
$S U(2)_{W}$ & $-19 / 6$ & $3 / 2$ \\
$U(1)_{Y}$ & $41 / 6$ & $27 / 2$ \\
\hline
\end{tabular}

This way, the shape of $\alpha_{4 i}(\mu)$ is determined as

$$
\alpha_{4 i}^{-1}(\mu)=\alpha_{4 i}^{-1}\left(m_{Z}\right)-\frac{\mathrm{b}_{i}^{\mathrm{SM}}}{2 \pi} \ln \frac{\mu}{m_{Z}}-\frac{\mathrm{b}_{i}^{6 \mathrm{D}}}{2 \pi} \sum_{l} \ln \frac{\mu}{m_{(l)}},
$$

where $m_{(l)}$ shows $l$ th KK mass and the upper bound of the summation is

$$
m_{(l)} \leq \mu .
$$

In the case of $S^{1}$ in five dimensions, where the spectrum of $\mathrm{KK}$ masses is equally spaced, the above calculation is executed with no difficulty. In $6 \mathrm{D}$ cases, by contrast, the KK mass spectrum is not equally spaced and we use the following approximation.

(i) $T^{2}$ case: In the $T^{2}$ case, the form of the $(m, n)$ th KK mass is $m_{(m, n)}=\sqrt{m^{2}+n^{2}} / R$ and eventually that of the first (lightest) $\mathrm{KK}$ mass is $M_{\mathrm{KK}}=1 / R$. The exact form of the summation is as follows:

$$
\sum_{(m, n)} \ln \frac{\mu}{\sqrt{m^{2}+n^{2}} M_{\mathrm{KK}}} \text { for } 1 \leq m^{2}+n^{2} \leq\left(\frac{\mu}{M_{\mathrm{KK}}}\right)^{2} .
$$

We approximate the summation over $m$ and $n$ by integral over $r$ and $\theta$ in two-dimensional polar coordinates:

$$
\begin{aligned}
& \sum_{(m, n)}\left(\ln \frac{\mu}{M_{\mathrm{KK}}}-\frac{1}{2} \ln \left(m^{2}+n^{2}\right)\right) \\
& \simeq \int_{1}^{\mu / M_{\mathrm{KK}}} 2 \pi d r \cdot r\left(\ln \frac{\mu}{M_{\mathrm{KK}}}-\ln r\right) \\
& =\frac{\pi}{2}\left[\left(\frac{\mu}{M_{\mathrm{KK}}}\right)^{2}-1-2 \ln \frac{\mu}{M_{\mathrm{KK}}}\right],
\end{aligned}
$$

which results in

$$
\begin{aligned}
\alpha_{4 i}^{-1}(\mu) \simeq & \alpha_{4 i}^{-1}\left(m_{Z}\right)-\frac{\mathrm{b}_{i}^{\mathrm{SM}}}{2 \pi} \ln \frac{\mu}{m_{Z}}-\frac{\mathrm{b}_{i}^{6 \mathrm{D}}}{2 \pi} \cdot \frac{\pi}{2} \\
& \times\left[\left(\frac{\mu}{M_{\mathrm{KK}}}\right)^{2}-1-2 \ln \frac{\mu}{M_{\mathrm{KK}}}\right] .
\end{aligned}
$$

(ii) $S^{2}$ case: In the $S^{2}$ case, the form of $(j, m)$ th KK mass is $m_{(j, m)}=\sqrt{j(j+1)} / R$ and that of the first (lightest) $\mathrm{KK}$ mass is $M_{\mathrm{KK}}=\sqrt{2} / R$. There are $2 j+1$ numbers of degenerated states in each $j$ level. The exact form of the summation is as follows:

$$
\begin{aligned}
& \sum_{j=1}^{j_{\max }}(2 j+1) \ln \left(\frac{\mu}{\sqrt{\frac{j(j+1)}{2}} M_{\mathrm{KK}}}\right) \\
& \text { for } 2 \leq j(j+1) \leq 2\left(\frac{\mu}{M_{\mathrm{KK}}}\right)^{2} .
\end{aligned}
$$

When we use the approximation: $j(j+1) \simeq j^{2}, j_{\max }$ and $j_{\min }$ are determined as

$$
j_{\max } \simeq \sqrt{2} \frac{\mu}{m_{\mathrm{KK}}}, \quad j_{\min } \simeq \sqrt{2} .
$$

Let us approximate the summation over $j$ by an integral over $j$ :

$$
\begin{aligned}
& \sum_{j=1}^{l_{\max }}(2 j+1) \ln \left(\frac{\mu}{\sqrt{\frac{j(j+1)}{2}} M_{\mathrm{KK}}}\right) \simeq \int_{j_{\min }}^{j_{\max }} d j(2 j) \ln \left(\frac{j_{\max }}{j}\right) \\
& =\left(\frac{\mu}{M_{\mathrm{KK}}}\right)^{2}-1-2 \ln \frac{\mu}{M_{\mathrm{KK}}} .
\end{aligned}
$$

Thereby we obtain the final form:

$$
\begin{aligned}
\alpha_{4 i}^{-1}(\mu) \simeq & \alpha_{4 i}^{-1}\left(m_{Z}\right)-\frac{\mathrm{b}_{i}^{S \mathrm{M}}}{2 \pi} \ln \left(\frac{\mu}{m_{\mathrm{Z}}}\right) \\
& -\frac{\mathrm{b}_{i}^{6 \mathrm{D}}}{2 \pi} \cdot 1\left[\left(\frac{\mu}{M_{\mathrm{KK}}}\right)^{2}-1-2 \ln \frac{\mu}{M_{\mathrm{KK}}}\right] .
\end{aligned}
$$

Combining Eq. (B8) and (B12), we get the final form (70). Neglecting the logarithmic terms in Eq. (70), we obtain

$$
\alpha_{4 i}^{-1}(\Lambda) \sim \alpha_{4 i}^{-1}\left(m_{Z}\right)-\frac{C b_{i}^{6 \mathrm{D}}}{2 \pi} \frac{\Lambda^{2}}{M_{\mathrm{KK}}^{2}} .
$$

We note that the coefficient of the quadratic term for $T^{2}$ coincides with that in Ref. [48] obtained from a different regularization scheme. Putting Eq. (B13) into the condition $\epsilon(\Lambda) \sim 1$, we get

$$
\Lambda^{2} \sim \frac{4 \pi M_{\mathrm{KK}}^{2}}{C\left(N_{g}+2 \mathrm{~b}_{i}^{6 \mathrm{D}}\right) \alpha_{4 i}\left(m_{Z}\right)},
$$

where we have used $V_{2}=8 \pi C / M_{\mathrm{KK}}^{2}$. Concretely, we get

$$
\Lambda \lesssim \begin{cases}5.3 M_{\mathrm{KK}} & \text { for } T^{2} \\ 6.6 M_{\mathrm{KK}} & \text { for } S^{2},\end{cases}
$$

from the $U(1)_{Y}$ cutoff.

In addition to this analysis, we also make a consideration for the Landau poles of the gauge interactions. If the value of the energy where a Landau pole emerges is smaller than that of the cutoff which we have discussed before, we should treat the position of the Landau pole, $\alpha_{4 i}^{-1}\left(\Lambda_{\text {Landau }}\right)=0$, which is easily obtained with leading order approximation as

$$
\Lambda_{\text {Landau }}^{2} \sim \frac{2 \pi M_{\mathrm{KK}}^{2}}{C \mathrm{~b}_{i}^{6 \mathrm{D}} \alpha_{4 i}\left(m_{Z}\right)},
$$


TABLE VI. The values of the cutoff scales and the positions of the Landau poles in $T^{2}$ and $S^{2}$ cases.

\begin{tabular}{lcc}
\hline \hline & \multicolumn{2}{c}{ Type of geometry } \\
\hline Types & $T^{2}$-based & $S^{2}$-based \\
$S U(3)_{C}$ cutoff & no cutoff & no cutoff \\
$S U(2)_{W}$ cutoff & $6.9 M_{\mathrm{KK}}$ & $8.6 M_{\mathrm{KK}}$ \\
$U(1)_{Y}$ cutoff & $5.3 M_{\mathrm{KK}}$ & $6.6 M_{\mathrm{KK}}$ \\
$S U(3)_{C}$ Landau pole & no cutoff & no cutoff \\
$S U(2)_{W}$ Landau pole & $8.9 M_{\mathrm{KK}}$ & $11 M_{\mathrm{KK}}$ \\
$U(1)_{Y}$ Landau pole & $5.4 M_{\mathrm{KK}}$ & $6.8 M_{\mathrm{KK}}$ \\
\hline \hline
\end{tabular}

as a cutoff scale instead. The concrete forms of each value are shown in Table VI.

In the analysis above, we have taken values of $N_{g}$ as 3,2, and 1 in each case of $S U(3)_{C}, S U(2)_{W}$ and $U(1)_{Y}$, respectively, and have employed the values,

$$
\begin{gathered}
\alpha_{U(1)_{Y}}^{-1}\left(m_{Z}\right)=97.9, \quad \alpha_{S U(2)_{W}}^{-1}\left(m_{Z}\right)=29.4, \\
\alpha_{S U(3)_{C}}^{-1}\left(m_{Z}\right)=8.44,
\end{gathered}
$$

at $m_{Z}=91.1 \mathrm{GeV}$. We do not consider a TeV-scale gauge coupling unification condition as a UV cutoff in this paper.

In both $T^{2}$ and $S^{2}$ cases, the most stringent bounds come from the $U(1)_{Y}$ cutoff scales, which restrict the effective range of the perturbation the most severely. It is natural that the scale emerging from the $U(1)_{Y}$ Landau pole is near the upper limit of the perturbativity but a little bit higher.

\section{APPENDIX C: BREIT-WIGNAR FORMULA}

We review how the Breit-Wigner formula emerges from the resummation of the one-particle irreducible (1PI) Higgs two-point function, in order to be careful of possible systematic errors when the width becomes broad. In this paper, we assume that the Higgs mass is larger than twice the $W \mathrm{KK}$ mass so that it is sufficient to limit ourselves to the SM case when discussing the Higgs total width. It is straightforward to extend the result for the UED when one wants to take the KK loops into account.

In the SM, the Higgs production cross section via the gluon fusion process is obtained as

$$
\hat{\sigma}_{g g \rightarrow H}=\frac{\pi^{2}}{8 M_{H}} \Gamma_{H \rightarrow g g}\left(M_{H}\right) \delta\left(\hat{s}-M_{H}^{2}\right),
$$

where

$$
\Gamma_{H \rightarrow g g}\left(M_{H}\right)=\frac{\alpha_{s}^{2}}{8 \pi^{3}} \frac{M_{H}^{3}}{v_{\mathrm{EW}}^{2}}\left|I\left(\frac{m_{t}^{2}}{M_{H}^{2}}\right)\right|^{2} .
$$

Then we get

$$
\begin{aligned}
\hat{\sigma}_{g g \rightarrow H \rightarrow Z Z} & \simeq \hat{\sigma}_{g g \rightarrow H} \operatorname{Br}_{H \rightarrow Z Z}\left(M_{H}\right) \\
& =\frac{\pi^{2}}{8 M_{H}} \Gamma_{H \rightarrow g g}\left(M_{H}\right) \mathrm{Br}_{H \rightarrow Z Z}\left(M_{H}\right) \delta\left(\hat{s}-M_{H}^{2}\right),
\end{aligned}
$$

where

$$
\begin{aligned}
\operatorname{Br}_{H \rightarrow Z Z}\left(M_{H}\right)= & \frac{\Gamma_{H \rightarrow Z Z}\left(M_{H}\right)}{\Gamma_{H}\left(M_{H}\right)} \\
= & \frac{\frac{M_{H}^{3}}{32 \pi v_{\mathrm{EW}}^{2}}\left[1-\frac{4 m_{Z}^{2}}{M_{H}^{2}}+\frac{12 m_{Z}^{4}}{M_{H}^{4}}\right] \sqrt{1-\frac{4 m_{Z}^{2}}{M_{H}^{2}}}}{\Gamma_{H}\left(M_{H}\right)} .
\end{aligned}
$$

The expression $(\mathrm{C} 1)$ is obtained in the limit of the vanishing decay width $\Gamma_{H} \rightarrow 0$. We may introduce a narrow width in Eq. (C3) by the Breit-Wigner type replacement,

$$
\delta\left(\hat{s}-M_{H}^{2}\right) \rightarrow \frac{1}{\pi} \frac{\Delta}{\left(\hat{s}-M_{H}^{2}\right)^{2}+\Delta^{2}},
$$

to get

$$
\begin{aligned}
\hat{\sigma}_{g g \rightarrow H \rightarrow Z Z} \simeq & \frac{\pi}{8 M_{H}} \frac{\Gamma_{H \rightarrow g g}\left(M_{H}\right) \Gamma_{H \rightarrow Z Z}\left(M_{H}\right)}{\Gamma_{H}\left(M_{H}\right)} \\
& \times \frac{\Delta}{\left(\hat{s}-M_{H}^{2}\right)^{2}+\Delta^{2}} .
\end{aligned}
$$

When we want to reproduce the delta function, $\Delta$ in Eq. (C5) cannot depend on $\hat{s}$, otherwise we cannot get the correct normalization: $\int d \hat{s} \delta\left(\hat{s}-M_{H}^{2}\right)=1$. One should then perform the replacement of the delta function (C5) as

$$
\Delta \rightarrow M_{H} \Gamma_{H}\left(M_{H}\right)
$$

in Eqs. (C1)-(C3). In the literature, see, e.g., [32]; $\Delta$ in Eq. (C6) is sometimes replaced as

$$
\Delta \rightarrow \frac{\hat{s}}{M_{H}} \Gamma_{H}\left(M_{H}\right) .
$$

Instead of the truncation (C3), we have already obtained the full $g g \rightarrow H \rightarrow Z Z$ cross section (50) by the naive Breit-Wignar type replacement $\Delta \rightarrow m_{H} \Gamma_{H}$ in and only in the denominator of the Higgs propagator,

$$
\frac{i}{Q^{2}-M_{H}^{2}+i \Delta} \text {. }
$$

Hereafter, let us see that this treatment gives a sufficiently good approximation to the full result (C28).

\section{Resummed propagator}

First let us review how the resummed propagator is obtained in the SM. We write the bare Higgs mass and field in terms of the renormalized ones and the counter terms as

$M_{B}^{2}=M_{H}^{2}+\delta M_{H}^{2}, \quad H_{B}=\sqrt{Z_{H}} H, \quad Z_{H}=1+\delta Z_{H}$.

The resummed bare propagator reads

$$
D_{B}=\frac{i}{Q^{2}-M_{B}^{2}+\Pi_{H}\left(Q^{2}\right)},
$$

where $M_{B}$ and $\Pi_{H}\left(Q^{2}\right)$ are the bare mass and the 1PI twopoint function, respectively, both of which contain UV divergences, and $Q^{2}:=-q^{2}$ for a Higgs four-momentum 
$q$. The renormalized propagator then becomes

$$
D_{R}=\frac{D_{B}}{Z_{H}}=\frac{i}{Q^{2}-M_{H}^{2}+\hat{\Sigma}_{H}\left(Q^{2}\right)},
$$

where

$$
\hat{\Sigma}_{H}\left(Q^{2}\right):=\Sigma_{H}\left(Q^{2}\right)-Z_{H} \delta M_{H}^{2}+\delta Z_{H}\left(Q^{2}-M_{H}^{2}\right)
$$

is the renormalized (finite) 1PI two-point function, with $\Sigma_{H}\left(Q^{2}\right):=Z_{H} \Pi_{H}\left(Q^{2}\right)$ being the 1PI two-point function that is given in terms of the renormalized fields but still contains UV divergences. Note that it is sufficient to consider the case $Q^{2}>0$ for our purpose since we have only $s$-channel Higgs propagator, though $Q^{2}$ can be negative when the Higgs is virtual, e.g., when exchanged in the $t$ channel. The Higgs two-point function in the SM is given by [71]

$$
\begin{aligned}
\Sigma_{H}\left(Q^{2}\right)= & -\frac{1}{16 \pi^{2} v^{2}}\left\{6 m_{t}^{2}\left[2 A_{0}\left(m_{t}^{2}\right)+\left(4 m_{t}^{2}-Q^{2}\right) B_{0}\left(Q^{2}, m_{t}^{2}\right)\right]-2\left[\left(6 m_{W}^{4}-2 Q^{2} m_{W}^{2}+\frac{M_{H}^{4}}{2}\right) B_{0}\left(Q^{2}, m_{W}^{2}\right)\right.\right. \\
& \left.+\left(3 m_{W}^{2}+\frac{M_{H}^{2}}{2}\right) A_{0}\left(m_{W}^{2}\right)-6 m_{W}^{4}\right]-\left[\left(6 m_{Z}^{4}-2 Q^{2} m_{Z}^{2}+\frac{M_{H}^{4}}{2}\right) B_{0}\left(Q^{2}, m_{Z}^{2}\right)+\left(3 m_{Z}^{2}+\frac{M_{H}^{2}}{2}\right) A_{0}\left(m_{Z}^{2}\right)-6 m_{Z}^{4}\right] \\
& \left.-\frac{3}{2}\left[3 M_{H}^{4} B_{0}\left(Q^{2}, M_{H}^{2}\right)+M_{H}^{2} A_{0}\left(M_{H}^{2}\right)\right]\right\},
\end{aligned}
$$

where the loop functions are

$$
A_{0}\left(m^{2}\right)=m^{2}\left(\Delta-\ln \frac{m^{2}}{\mu^{2}}+1\right),
$$

with $\Delta:=\frac{2}{\epsilon}-\gamma+\ln 4 \pi$ for $D=4-\epsilon$, and

$$
B_{0}\left(Q^{2}, m^{2}\right)=\Delta-\int_{0}^{1} d x \ln \frac{Q^{2} x(x-1)+m^{2}-i \varepsilon}{\mu^{2}}=\Delta-\ln \frac{m^{2}}{\mu^{2}}+I\left(\frac{4 m^{2}}{Q^{2}}\right),
$$

where

$$
I(\kappa):=\int_{0}^{1} d x \frac{x(2 x-1)}{\left(x-\frac{1}{2}\right)^{2}-\frac{1-\kappa}{4}-i \varepsilon}=\int_{0}^{1} d x \frac{2 x^{2}-x}{x^{2}-x+\frac{\kappa}{4}-i \varepsilon} .
$$

For concreteness we write down

$$
\begin{gathered}
I(\kappa)= \begin{cases}2\left(1-\sqrt{\kappa-1} \arctan \left(\frac{1}{\sqrt{\kappa-1}}\right)\right) & (\kappa \geq 1 \text { or } \operatorname{Im} \kappa \neq 0) \\
2-\sqrt{1-\kappa} \ln \left(\frac{1+\sqrt{1-\kappa}}{1-\sqrt{1-\kappa}}\right)+i \pi \sqrt{1-\kappa} & (0<\kappa \leq 1),\end{cases} \\
I^{\prime}(\kappa)= \begin{cases}\frac{1}{\kappa}-\frac{\arctan \left(\frac{1}{\sqrt{\kappa-1}}\right)}{\sqrt{\kappa-1}} & (\kappa>1 \text { or Im } \kappa \neq 0) \\
\frac{1}{\kappa}+\frac{1}{2 \sqrt{1-\kappa}} \ln \left(\frac{1+\sqrt{1-\kappa}}{1-\sqrt{1-\kappa}}\right)-\frac{i \pi}{2 \sqrt{1-\kappa}} & (0<\kappa<1) .\end{cases}
\end{gathered}
$$

Note that when $\operatorname{Im} \kappa=0$,

$$
\operatorname{Im} I(\kappa)=\pi \sqrt{1-\kappa} \theta(1-\kappa), \quad \operatorname{Im} I^{\prime}(\kappa)=-\frac{\pi}{2 \sqrt{1-\kappa}} \theta(1-\kappa) .
$$

In particular,

$$
I(4)=\frac{6-\sqrt{3} \pi}{3} \simeq 0.18, \quad I^{\prime}(4)=-\frac{2 \sqrt{3} \pi-9}{36} \simeq-0.052,
$$

and for small and large $\kappa$,

$$
\begin{gathered}
I(\kappa)=2+\ln \frac{\kappa}{4}+i \pi+O(\kappa \ln \kappa) \quad(\kappa \ll 1), \\
I(\kappa)=\frac{2}{3 \kappa}+O\left(\kappa^{-2}\right) \quad(\kappa \gg 1) .
\end{gathered}
$$




\section{On-shell scheme renormalization}

In the on-shell scheme, we put the following renormalization conditions:

$$
\left.\operatorname{Re} \hat{\Sigma}_{H}\left(Q^{2}\right)\right|_{Q^{2}=M_{H}^{2}}=0,\left.\quad \operatorname{Re} \frac{\partial}{\partial Q^{2}} \hat{\Sigma}_{H}\left(Q^{2}\right)\right|_{Q^{2}=M_{H}^{2}}=0,
$$

which gives

$$
\begin{aligned}
\hat{\Sigma}_{H}\left(Q^{2}\right)= & \Sigma_{H}\left(Q^{2}\right)-\operatorname{Re} \Sigma_{H}\left(M_{H}^{2}\right)-\operatorname{Re} \Sigma_{H}^{\prime}\left(M_{H}^{2}\right)\left(Q^{2}-M_{H}^{2}\right) \\
= & -\frac{1}{16 \pi^{2} v^{2}}\left(6 m_{t}^{2}\left\{\left(4 m_{t}^{2}-Q^{2}\right)\left[I\left(\frac{4 m_{t}^{2}}{Q^{2}}\right)-\operatorname{Re} I\left(\frac{4 m_{t}^{2}}{M_{H}^{2}}\right)\right]+\left(4 m_{t}^{2}-M_{H}^{2}\right) \frac{4 m_{t}^{2}}{M_{H}^{4}} \operatorname{Re} I^{\prime}\left(\frac{4 m_{t}^{2}}{M_{H}^{2}}\right)\left(Q^{2}-M_{H}^{2}\right)\right\}\right. \\
& -2\left\{\left(6 m_{W}^{4}-2 Q^{2} m_{W}^{2}+\frac{M_{H}^{4}}{2}\right)\left[I\left(\frac{4 m_{W}^{2}}{Q^{2}}\right)-\operatorname{Re} I\left(\frac{4 m_{W}^{2}}{M_{H}^{2}}\right)\right]+\left(6 m_{W}^{4}-2 M_{H}^{2} m_{W}^{2}+\frac{M_{H}^{4}}{2}\right)\right. \\
& \left.\times \frac{4 m_{W}^{2}}{M_{H}^{4}} \operatorname{Re} I^{\prime}\left(\frac{4 m_{W}^{2}}{M_{H}^{2}}\right)\left(Q^{2}-M_{H}^{2}\right)\right\}-\left\{\left(6 m_{Z}^{4}-2 Q^{2} m_{Z}^{2}+\frac{M_{H}^{4}}{2}\right)\left[I\left(\frac{4 m_{Z}^{2}}{Q^{2}}\right)-\operatorname{Re} I\left(\frac{4 m_{Z}^{2}}{M_{H}^{2}}\right)\right]\right. \\
& \left.\left.+\left(6 m_{Z}^{4}-2 M_{H}^{2} m_{Z}^{2}+\frac{M_{H}^{4}}{2}\right) \frac{4 m_{Z}^{2}}{M_{H}^{4}} \operatorname{Re} I^{\prime}\left(\frac{4 m_{Z}^{2}}{M_{H}^{2}}\right)\left(Q^{2}-M_{H}^{2}\right)\right\}-\frac{9 M_{H}^{4}}{2}\left\{\left[I\left(\frac{4 M_{H}^{2}}{Q^{2}}\right)-I(4)\right]+\frac{4}{M_{H}^{2}} I^{\prime}(4)\left(Q^{2}-M_{H}^{2}\right)\right\}\right),
\end{aligned}
$$

and hence,

$$
\begin{aligned}
\operatorname{Im} \hat{\Sigma}_{H}\left(Q^{2}\right)= & -\frac{1}{16 \pi^{2} v^{2}}\left\{-6 m_{t}^{2} Q^{2} \pi\left[1-\frac{4 m_{t}^{2}}{Q^{2}}\right]^{3 / 2} \theta\left(1-\frac{4 m_{t}^{2}}{Q^{2}}\right)-M_{H}^{4}\left(\frac{12 m_{W}^{4}}{M_{H}^{4}}-\frac{4 Q^{2} m_{W}^{2}}{M_{H}^{4}}+1\right) \pi \sqrt{1-\frac{4 m_{W}^{2}}{Q^{2}}} \theta\left(1-\frac{4 m_{W}^{2}}{Q^{2}}\right)\right. \\
& \left.-\frac{M_{H}^{4}}{2}\left(\frac{12 m_{Z}^{4}}{M_{H}^{4}}-\frac{4 Q^{2} m_{Z}^{2}}{M_{H}^{4}}+1\right) \pi \sqrt{1-\frac{4 m_{Z}^{2}}{Q^{2}}} \theta\left(1-\frac{4 m_{Z}^{2}}{Q^{2}}\right)-\frac{9 M_{H}^{4}}{2} \pi \sqrt{1-\frac{4 M_{H}^{2}}{Q^{2}}} \theta\left(1-\frac{4 M_{H}^{2}}{Q^{2}}\right)\right\} .
\end{aligned}
$$

We can compare this result with the tree-level Higgs decay width,

$$
\begin{aligned}
\Gamma_{H}^{\mathrm{tree}}\left(M_{H}\right)= & \frac{M_{H}^{3}}{16 \pi v_{\mathrm{EW}}^{2}}\left[1-\frac{4 m_{W}^{2}}{M_{H}^{2}}+\frac{12 m_{W}^{4}}{M_{H}^{4}}\right] \sqrt{1-\frac{4 m_{W}^{2}}{M_{H}^{2}}} \theta\left(M_{H}-2 m_{W}\right) \\
& +\frac{M_{H}^{3}}{32 \pi v_{\mathrm{EW}}^{2}}\left[1-\frac{4 m_{Z}^{2}}{M_{H}^{2}}+\frac{12 m_{Z}^{4}}{M_{H}^{4}}\right] \sqrt{1-\frac{4 m_{Z}^{2}}{M_{H}^{2}}} \theta\left(M_{H}-2 m_{Z}\right)+\frac{3 M_{H} m_{t}^{2}}{8 \pi v_{\mathrm{EW}}^{2}}\left[1-\frac{4 m_{t}^{2}}{M_{H}^{2}}\right]^{3 / 2} \theta\left(M_{H}-2 m_{t}\right) .
\end{aligned}
$$

We see from Eq. (C16) that the leading term for the $Q \sim M_{H} \gg 2 m_{t}$ limit is not proportional to $Q^{2}$ for the $W$ and $Z$ contributions and therefore the replacement (C12), namely,

$$
D_{R} \rightarrow \frac{i}{Q^{2}-M_{H}^{2}+i \frac{Q^{2}}{M_{H}} \Gamma_{H}}
$$

does not give a good fit.

When computing the full cross section for the process $g g \rightarrow H \rightarrow Z Z$, we may employ the resummed propagator (C12). Neglecting the contributions from box diagrams, we get the cross section,

$$
\hat{\sigma}_{g g \rightarrow H \rightarrow Z Z}=\frac{\alpha_{s}^{2}}{256 \pi^{2}}\left(\frac{m_{Z}}{v_{\mathrm{EW}}}\right)^{4}\left[1+\frac{\left(\hat{s}-2 m_{Z}^{2}\right)^{2}}{8 m_{Z}^{4}}\right] \sqrt{1-\frac{4 m_{Z}^{2}}{\hat{s}}}\left|I\left(\frac{m_{t}^{2}}{\hat{s}}\right)\right|^{2} \frac{1}{\pi} \frac{\hat{s}}{\left(\hat{s}-M_{H}^{2}+\operatorname{Re} \hat{\Sigma}_{H}(\hat{s})\right)^{2}+\left(\operatorname{Im} \hat{\Sigma}_{H}(\hat{s})\right)^{2}} .
$$

In Fig. 7, we show the results for various replacements. We see that any replacement suffices when the Higgs mass is not very large, $M_{H}=300 \mathrm{GeV}$ (left). (This is the case for the Higgs mass above the top threshold $m_{H} \gtrsim 2 m_{t}$ too.) For the large Higgs mass (right), the decay width becomes larger and we see that our approximation (50) with a
Breit-Wignar type replacement in the denominator $\Delta \rightarrow M_{H} \Gamma_{H}$ gives a good fit to the cross section with the full two-point function (C29).

Finally, just for comparison with Eq. (C6), let us present the resummed cross section (C29) in a rewritten form, 

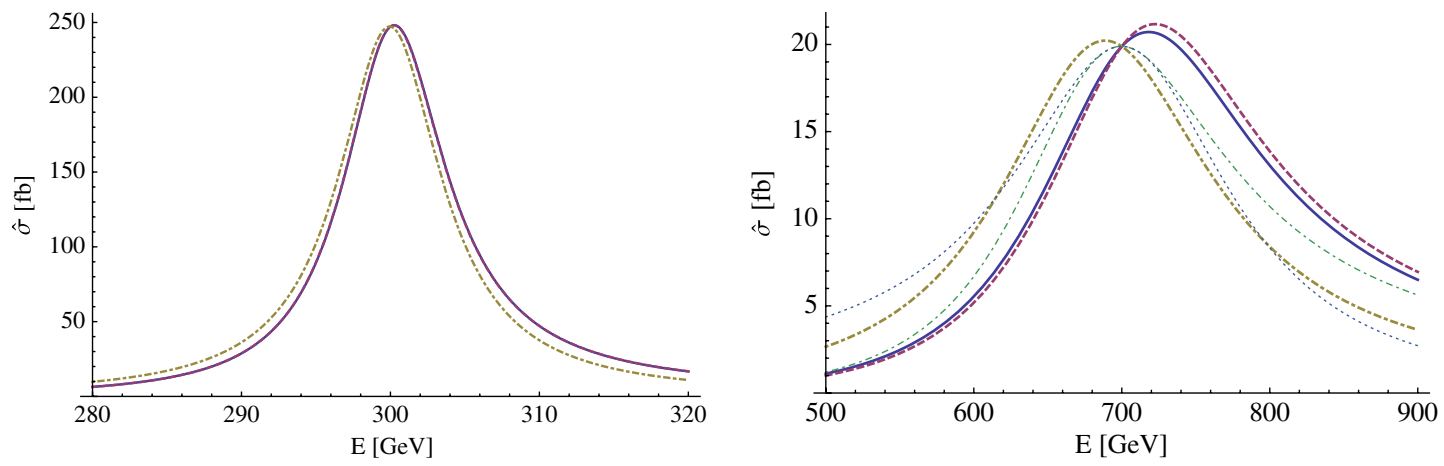

FIG. 7 (color online). Parton-level cross section for the process $g g \rightarrow H \rightarrow Z Z$ with $m_{H}=300 \mathrm{GeV}$ (left) and $700 \mathrm{GeV}$ (right). The inclusion of a full two-point function (C28) (blue solid line), the full one (50) with $\Delta \rightarrow M_{H} \Gamma_{H}$ (red dashed), and the truncated one (C6) with $\Delta \rightarrow \hat{s} \Gamma_{H} / M_{H}$ (yellow dot-dashed) are shown. In the right figure, the full one (50) with $\Delta \rightarrow \hat{s} \Gamma_{H} / M_{H}$ (thin dot-dashed) and the truncated one (C6) with $\Delta \rightarrow M_{H} \Gamma_{H}$ (thin dotted) are also drawn.

$$
\hat{\sigma}_{g g \rightarrow H \rightarrow Z Z}=\frac{\pi}{8 M_{H}} \frac{\alpha_{s}^{2} M_{H}^{3}}{8 \pi^{3} v_{\mathrm{EW}}^{2}}\left|\frac{\hat{s}}{M_{H}^{2}} I\left(\frac{m_{t}^{2}}{\hat{s}}\right)\right|^{2} \frac{\frac{\hat{s}}{M_{H}} \Gamma_{H}\left(M_{H}\right)}{\left(\hat{s}-M_{H}^{2}+\operatorname{Re}_{H}(\hat{s})\right)^{2}+\left(\operatorname{Im}_{H}(\hat{s})\right)^{2}} \frac{\frac{M_{H}^{3}}{32 \pi v_{\mathrm{EW}}^{2}}\left[1-\frac{4 m_{Z}^{2}}{\hat{s}}+\frac{12 m_{Z}^{4}}{\hat{s}^{2}}\right] \sqrt{1-\frac{4 m_{Z}^{2}}{\hat{s}}}}{\Gamma_{H}\left(M_{H}\right)} .
$$

\section{Pole-scheme renormalization}

Let us review the pole-scheme renormalization [72]. Instead of the on-shell condition (C24), the pole-scheme renormalization condition fixes the pole of the propagator (C11) at $\bar{Q}^{2}$, which we parametrize by two real constants $m_{H}$ and $\Gamma_{H}$ as $\bar{Q}^{2}=m_{H}^{2}-i m_{H} \Gamma_{H}$, namely,

$$
\bar{Q}^{2}-M_{B}^{2}+\Pi_{H}\left(\bar{Q}^{2}\right)=0 .
$$

As the pole position of the bare propagator $(\mathrm{C} 11)$ is the same as that of the renormalized one (C12), we see that the on-shell renormalized two-point function satisfies

$$
\bar{Q}^{2}-M_{H}^{2}+\hat{\Sigma}_{H}\left(\bar{Q}^{2}\right)=0,
$$

that is,

$$
M_{H}^{2}=m_{H}^{2}+\operatorname{Re} \hat{\Sigma}_{H}\left(\bar{Q}^{2}\right), \quad m_{H} \Gamma_{H}=\operatorname{Im} \hat{\Sigma}_{H}\left(\bar{Q}^{2}\right) .
$$
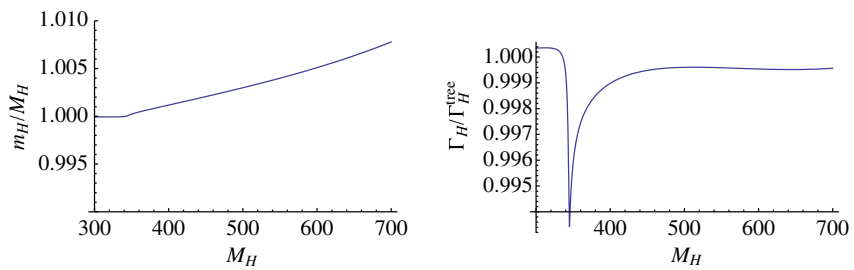

FIG. 8 (color online). Ratio of the pole mass $m_{H}$ to the onshell one $M_{H}$ as a function of the on-shell mass (left); same for the ratio of the pole-scheme width $\Gamma_{H}$ to the tree-level one $\Gamma_{H}^{\text {tree }}$ (right).
We see that the real and imaginary parts determine the pole-scheme mass $m_{H}$ and decay rate $\Gamma_{H}$ as functions of the on-shell scheme mass $M_{H}$. Note that the renormalized two-point function $\hat{\Sigma}_{H}$ has an implicit dependence on the on-shell mass $M_{H}$.

In Fig. 8, we plot the ratio of the pole to on-shell mass $m_{H} / M_{H}$ (left) and the pole-scheme width $\Gamma_{H}$ to the treelevel width (C27) (right) as functions of the on-shell mass $M_{H}$, computed within the SM.

It is known that the on-shell scheme mass is gauge dependent at the next-to-next-to-leading order; see references in [72]. In contrast, the pole position of the amplitude is a gauge independent physical notion. Therefore, in principle, we should utilize the polescheme mass and width. However, the on-shell mass and the pole mass are identical at consistent 1-loop order (which is the highest order considered here), and they start differing only at 2-loop order and above. We see from Fig. 8 that both schemes agree within $1 \%$ accuracy in our approximated treatment neglecting nonresonant box contributions, which are the same order as $O(\alpha)$ corrections to the resonant ones. ${ }^{13}$ We safely utilize the

\footnotetext{
${ }^{13}$ The difference in Fig. 8 stems from the fact that a complex value is inserted for $\bar{Q}^{2}$ in Eq. (C33), i.e., $\bar{Q}^{2}=m_{H}^{2}-i m_{H} \Gamma_{H}$. However, $\Gamma_{H}$ is formally a higher-order term, since it corresponds to the imaginary part of the self-energy, which first occurs at one-loop order. Therefore, when inserted into the one-loop self-energies in Eq. (C33), this leads to a contribution that is formally at the next-to-next-to-leading order level, which causes the small numerical difference between the two schemes in Fig. 8.
} 
on-shell mass and the tree-level width (C27) even though the Higgs decay width becomes as large as $180 \mathrm{GeV}$ when $M_{H}=700 \mathrm{GeV}$.

Note that our treatment to include the decay width in the numerical calculation (50) with (54) corresponds to the (gauge invariant) complex-mass scheme; see, e.g., Refs. [73,74], where the real Higgs mass is replaced by a complex value everywhere in the amplitude, which in our case leads to a replacement of the Higgs mass in its propagator only.
[1] T. Appelquist, H.-C. Cheng, and B. A. Dobrescu, Phys. Rev. D 64, 035002 (2001).

[2] G. Servant and T.M.P. Tait, Nucl. Phys. B650, 391 (2003).

[3] G. Bhattacharyya, A. Datta, S. K. Majee, and A. Raychaudhuri, Nucl. Phys. B760, 117 (2007).

[4] G. D. Kribs, arXiv:hep-ph/0605325.

[5] D. Hooper and S. Profumo, Phys. Rep. 453, 29 (2007).

[6] G. Belanger, M. Kakizaki, and A. Pukhov, arXiv:1012.2577.

[7] H.-C. Cheng, K. T. Matchev, and M. Schmaltz, Phys. Rev. D 66, 036005 (2002).

[8] H.-C. Cheng, K. T. Matchev, and M. Schmaltz, Phys. Rev. D 66, 056006 (2002).

[9] C. Macesanu, C. McMullen, and S. Nandi, Phys. Rev. D 66, 015009 (2002).

[10] M. Kazana, Acta Phys. Pol. B 38, 449 (2007).

[11] G. Bhattacharyya, A. Datta, S. K. Majee, and A. Raychaudhuri, Nucl. Phys. B821, 48 (2009).

[12] D. Choudhury, A. Datta, and K. Ghosh, J. High Energy Phys. 08 (2010) 051.

[13] B. Bhattacherjee and K. Ghosh, Phys. Rev. D 83, 034003 (2011).

[14] H. Murayama, M. Nojiri, and K. Tobioka, arXiv:1107.3369v2.

[15] G. Burdman, B. A. Dobrescu, and E. Ponton, Phys. Rev. D 74, 075008 (2006).

[16] B. A. Dobrescu, K. Kong, and R. Mahbubani, J. High Energy Phys. 07 (2007) 006.

[17] K. Ghosh, J. High Energy Phys. 04 (2009) 049.

[18] K. Ghosh and A. Datta, Nucl. Phys. B800, 109 (2008).

[19] G. Bertone, C. Jackson, G. Shaughnessy, T. M. Tait, and A. Vallinotto, Phys. Rev. D 80, 023512 (2009).

[20] G. Cacciapaglia, A. Deandrea, and J. Llodra-Perez, J. High Energy Phys. 10 (2011) 146.

[21] G. Cacciapaglia et al., J. High Energy Phys. 10 (2011) 042.

[22] G. Bhattacharyya, P. Dey, A. Kundu, and A. Raychaudhuri, Phys. Lett. B 628, 141 (2005).

[23] B. Bhattacherjee and A. Kundu, Phys. Lett. B 627, 137 (2005).

[24] A. Freitas and K. Kong, J. High Energy Phys. 02 (2008) 068.

[25] K. Ghosh and A. Datta, Phys. Lett. B 665, 369 (2008).

[26] M. Battaglia, A. Datta, A. De Roeck, K. Kong, and K. T. Matchev, J. High Energy Phys. 07 (2005) 033.

[27] B. Bhattacherjee, A. Kundu, S. K. Rai, and S. Raychaudhuri, Phys. Rev. D 78, 115005 (2008).

[28] K. Tobioka, Master thesis, University of Tokyo, 2011.
[29] U. Haisch and A. Weiler, Phys. Rev. D 76, 034014 (2007).

[30] A. Freitas and U. Haisch, Phys. Rev. D 77, 093008 (2008).

[31] G. Aad et al. (ATLAS Collaboration), Eur. Phys. J. C 71, 1744 (2011)

[32] A. Djouadi, Phys. Rep. 457, 1 (2008).

[33] S. Riemann, Rep. Prog. Phys. 73, 126201 (2010).

[34] T. Appelquist and H.-U. Yee, Phys. Rev. D 67, 055002 (2003).

[35] I. Gogoladze and C. Macesanu, Phys. Rev. D 74, 093012 (2006).

[36] M. Baak et al., arXiv:1107.0975.

[37] F. J. Petriello, J. High Energy Phys. 05 (2002) 003.

[38] N. Maru, T. Nomura, J. Sato, and M. Yamanaka, Eur. Phys. J. C 66, 283 (2010).

[39] K. Nishiwaki, arXiv:1101.0649.

[40] N. Haba, K. Oda, and R. Takahashi, Acta Phys. Pol. B 42, 33 (2011).

[41] K. Nishiwaki and K.-y. Oda, Eur. Phys. J. C 71, 1786 (2011).

[42] R. N. Mohapatra and A. Perez-Lorenzana, Phys. Rev. D 67, 075015 (2003).

[43] B. A. Dobrescu and E. Ponton, J. High Energy Phys. 03 (2004) 071.

[44] G. Burdman, B. A. Dobrescu, and E. Ponton, J. High Energy Phys. 02, 033 (2006).

[45] G. Cacciapaglia, A. Deandrea, and J. Llodra-Perez, J. High Energy Phys. 03, 083 (2010).

[46] H. Dohi and K.-y. Oda, Phys. Lett. B 692, 114 (2010).

[47] N. Maru, T. Nomura, J. Sato, and M. Yamanaka, Nucl. Phys. B830, 414 (2010).

[48] K. R. Dienes, E. Dudas, and T. Gherghetta, Nucl. Phys. B537, 47 (1999).

[49] S. Weinberg, The Quantum Theory of Fields. Vol. 1: Foundations (Cambridge University Press, Cambridge, 1995), p. 609.

[50] C. S. Lim, N. Maru, and K. Nishiwaki, Phys. Rev. D 81, 076006 (2010).

[51] B. A. Dobrescu and E. Poppitz, Phys. Rev. Lett. 87, 031801 (2001).

[52] E. Ponton and L. Wang, J. High Energy Phys. 11 (2006) 018.

[53] A. T. Azatov, J. High Energy Phys. 10 (2007) 067.

[54] S. Randjbar-Daemi, A. Salam, and J. A. Strathdee, Nucl. Phys. B214, 491 (1983).

[55] E. C. G. Stueckelberg, Helv. Phys. Acta 11, 225 (1938).

[56] V.I. Ogievetskii and I. V. Polubarinov, Soviet Physics JETP 14, 179 (1962).

[57] H. Ruegg and M. Ruiz-Altaba, Int. J. Mod. Phys. A 19, 3265 (2004). 
[58] B. Kors and P. Nath, J. High Energy Phys. 07 (2005) 069.

[59] H. Georgi, A. K. Grant, and G. Hailu, Phys. Lett. B 506, 207 (2001).

[60] H. M. Georgi, S.L. Glashow, M. E. Machacek, and D. V. Nanopoulos, Phys. Rev. Lett. 40, 692 (1978).

[61] E. Glover and J. van der Bij, Nucl. Phys. B321, 561 (1989).

[62] A. Djouadi, Phys. Rep. 459, 1 (2008).

[63] H. L. Lai et al. (CTEQ), Eur. Phys. J. C 12, 375 (2000).

[64] M. Bauce et al., http://www-cdf.fnal.gov/physics/ewk/ 2009/ZZ1111/ZZWeb/.

[65] V. M. Abazov et al. (D0 Collaboration), Phys. Rev. D 84, 011103(R) (2011).

[66] A. Robson, Europhysics Conference on High-Energy Physics, Grenoble, France, 2011 (unpublished); see also http://eps-hep2011.eu/.
[67] K. Cranmer, Europhysics Conference on High-Energy Physics, Grenoble, France, 2011 (unpublished); see also http://eps-hep2011.eu/.

[68] A. Korytov, Europhysics Conference on High-Energy Physics, Grenoble, France, 2011 (unpublished); see also http://eps-hep2011.eu/.

[69] K. Nishiwaki, K.-y. Oda, N. Okuda, and R. Watanabe, arXiv:1108.1764.

[70] J. Steinberger, Phys. Rev. 76, 1180 (1949).

[71] A. Denner, Fortschr. Phys. 41, 307 (1993).

[72] B. A. Kniehl and A. Sirlin, Phys. Lett. B 530, 129 (2002).

[73] A. Denner, S. Dittmaier, M. Roth, and D. Wackeroth, Nucl. Phys. B560, 33 (1999).

[74] A. Denner, S. Dittmaier, M. Roth, and L. H. Wieders, Nucl. Phys. B724, 247 (2005). 